\title{
ON DETECTING OPEN COLLARS
}

\author{
BY \\ L. C. SIEBENMANN(!)
}

0. Introduction. When is a manifold $W$ isomorphic to an open collar of its boundary-i.e. when is $W \cong b W \times[0,1)$ ? One would hope for a convenient homotopy criterion at least in these categories of (metrizable, finite-dimensional) manifolds:

$$
\begin{aligned}
\text { DIFF } & =\text { smooth (i.e. differentiable } C^{\infty} \text { ) manifolds and smooth maps. } \\
\text { PL } & =\text { piecewise linear manifolds and piecewise linear maps. } \\
\text { TOP } & =\text { topological manifolds and continuous maps. }
\end{aligned}
$$

In TOP we have no success $\left({ }^{2}\right)$; but for DIFF and PL manifolds of dimension $\geqq 5$ we obtain roughly stated, the following:

(Open Collar Theorem 1.6). $W \cong b W \times[0,1)$ if and only if the inclusion $b W \subseteq W$ is a homotopy equivalence and the system of fundamental groups of complements of compacta in $W$ behaves algebraically like that in $b W \times[0,1)$. Corollary. $W \cong b W$ $\times[0,1)$ if and only if the pairs $(W, b W)$ and $(b W \times[0,1), b W \times 0)$ are proper homotopy equivalent $\left({ }^{3}\right)$.

Notice that $b W$ need not be compact.

In the proof, the fundamental group condition ( $\pi_{1}$ condition) permits imbedded surgery with handles of dimension $\leqq 2$ to find large compacta $K \subset W$ such that 2-connectivity of $(W, W-K)$ can be proved. Poincare duality in the universal covering seems essential to show this. A well-known engulfing argument of Stallings completes the proof. (We first encountered a proof of this result for $n \geqq 6$ based entirely on handlebody theory cf. [40].) Although the $\pi_{1}$ condition requires careful statement, our applications show that it is pleasant to use. Indeed the main contention of this paper is that it considerably extends the usefulness of Stallings' conception of engulfing.

That the $\pi_{1}$ condition is necessary at all (i.e. not redundant) is at first sight surprising. This was first noticed in the wake of J. H. C. Whitehead's misproof of the 3-dimensional Poincaré conjecture in 1934. Whitehead then constructed a

Received by the editors May 8, 1967.

(1) The author was supported during a part of this work by the National Research Council of Canada. article.

$\left.{ }^{2}\right)$ The same result for TOP is now established. See note added in proof at the end of this

$\left(^{3}\right)$ That is, homotopy equivalent in the subcategory with the same objects but only proper maps. A map is proper if the preimage of each compact set is compact. 
contractible open subset $U$ of $R^{3}$ not homeomorphic to $R^{3}$ thus disposing of the Poincaré conjecture for open 3-manifolds. If $W^{3}=U-B^{3}$, where $B^{3}$ is interior of a small ball in $U$, then $b W \subseteq W$ is a homotopy equivalence, but Whitehead and Newman showed in [36] that the $\pi_{1}$ condition is violated. All products $W \times M$, $M$ a closed manifold, must also violate the $\pi_{1}$ condition. For examples arising from higher dimensional contractible manifolds see [37], [54].

The Open Collar Theorem immediately gives a homotopy characterization of open regular neighbourhoods of subcomplexes of codimension $\geqq 3$ in DIFF or PL manifolds of dimension $\geqq 5$. (See $\$ 2.1$ for a precise statement.) In DIFF one gets a characterization of open tubular neighbourhoods (\$2.2). These are more general than parallel known characterizations of closed regular (or tubular) neighbourhoods [24] via the $s$-cobordism theorem in that noncompact subobjects are treated. There follows ( $\$ 2.3)$ a pleasant proof of the stable isomorphism theorem (in relative form) for possibly noncompact manifolds of any category.

It states: Let $f:\left(M_{1}, b M_{1}\right) \rightarrow\left(M_{2}, b M_{2}\right)$ be a (pair) homotopy equivalence of manifolds (with boundary) of the same dimension. If the tangent bundle $\tau\left(M_{1}\right)$ is isomorphic to $f^{*} \tau\left(M_{2}\right)$ as a stable bundle (or microbundle) in the given category, then there exists an euclidean space $R^{n}$ and an isomorphism $M_{1} \times R^{n} \cong M_{2} \times R^{n}$ (pair) homotopic to $f \times\left(1 \mid R^{n}\right)$. B. Mazur, who formulated this result and proved it for closed DIFF manifolds, has already presented a proof in this generality [26]. It is very different, rather complicated, and seems to contain a gap $\left({ }^{4}\right)$. $\mathrm{M}$. Hirsch has given a proof valid if $f$ is a proper homotopy equivalence. The simplest case not covered by Hirsch's proof has $M_{1}=S^{1} \times S^{1}$-(point) i.e. the punctured torus, $M_{2}=R^{2}-$ (two points), and $f$ any homotopy equivalence. Since $M_{1}$ contains arbitrarily large compact sets with connected complement and $M_{2}$ does not, $f$ cannot be a proper homotopy equivalence. Yet visibly $M_{1} \times R^{1} \cong M_{2} \times R^{1}$ is a doughnut with two holes!

The stable isomorphism theorem leads one to ask when a tangential homotopy equivalence (like $f$ above) can itself be deformed to an isomorphism. For DIFF and PL manifolds, without boundary, of dimension $n \geqq 5$, it suffices that $M_{1}$ and $M_{2}$ be open regular neighborhoods of complexes of dimension $<n / 2$. This will be proved in [41] (also in a relative form), by use of the Open Collar Theorem in combination with a sort of covering homotopy theorem for regular neighborhoods.

D. B. A. Epstein suggested

THeorem 2.7. Suppose $X$ is an oriented unbounded DIFF or PL n-manifold $n \geqq 5$. If there exists a degree 1 proper map $R^{n} \rightarrow X$, then $X \cong R^{n}$.

The next application is

THEOREM 3.1 (FOR DIFF). Let $\alpha: G \times W \rightarrow W$ be a smooth action of a compact Lie group $G$ on a connected unbounded DIFF manifold $W$. Suppose the fixed point

$\left.{ }^{4}\right)$ In [26] the proof of Proposition 3 begins with an untrue assertion. Then the second diagram on page 392 of [26] is not commutative. 
set $M$ is a submanifold so situated that $W$ is an open tubular neighbourhood of $M$. (See $\$ 2.2$ for a test), and suppose that the isotropy subgroups $G_{x}$ for $x \in W-M$ are all conjugate. (By definition $G_{x}=\{g \in G \mid \alpha(g, x)=x\}$.) Then, provided the orbit space of $\alpha$ on $W$ has dimension $\geqq 5, W$ admits the structure of a smooth euclidean vector bundle over $M$ such that $\alpha$ respects fibers and acts orthogonally on each fiber.

The case $(W, M)=\left(R^{n}, R^{k}\right)$ together with $\S 2.2$ mends the fault in the engulfing argument of Connell, Montgomery and Yang [4] noted in [5]. The remedy intended in [5] is different (as Connell has told us) and appears not to establish Theorem 3.1. We compare the situation for actions on closed tubes, which is complicated by simple homotopy type.

The last application affirms a conjecture of J. P. Hempel and D. R. McMillan [12] as follows:

THEOREM 4.1. Suppose $\Sigma^{k}$ is a $k$-sphere topologically, (but possibly wildly) imbedded in the $n$-sphere $S^{n}, n \geqq 5, n-k \geqq 3$, so that $S^{n}-\Sigma^{k}$ is $1-\mathrm{LC}$ at each point of $\Sigma^{k}$. Then $S^{n}-\Sigma^{k}$ is homeomorphic (indeed diffeomorphic) to $S^{n}-S^{k}$, where, $S^{k} \subset S^{n}$ is the standard unknotted $k$-sphere.

For more discussion of this see $\$ 4$.

The sections to come are as follows:

$\$ 1$ The open collar theorem

\$2 Detecting open regular neighborhoods (includes stable isomorphism and Epstein's application)

\$3 Application to group actions (with contrasting results about actions on closed tubes)

$\S 4$ Unknotting complements of spheres

\section{The open collar theorem.}

Proposition 1.1. If $W$ is a connected topological manifold with boundary such that $\pi_{*}(W, b W)=0$, then $W$ is noncompact with exactly one end-i.e. for $K$ compact in $W, W-K$ has exactly one unbounded component.

We will deduce this from a considerably more general proposition.

Definitions. Let $W$ be a topological manifold. We say that $W$ has $\geqq k$ ends if there exist compact sets $K \subset W$ so that $W-K$ has $\geqq k$ unbounded( $\left(^{5}\right)$ components. We say $W$ has exactly $k$ ends if it has $\geqq k$ ends but not $\geqq k+1$ and we say $W$ has $\infty$ ends if it has $\geqq k$ ends for all finite $k$.

Proposition 1.2. Let $W^{n}$ be a connected topological n-manifold with boundary $b W$. Let $e$ be the number of ends of $W$ (e finite or $\infty)$, and let $Z^{t}$ be the bundle of

(5) An unbounded set is one with noncompact closure. 
twisted integer coefficients over $W$ corresponding to the orientation homomorphism $\pi_{1}(W) \rightarrow\{ \pm 1\}$. Then

$$
\operatorname{rank}\left\{H_{n-1}\left(W, b W ; Z^{t}\right)\right\}+1 \geqq e .
$$

Proof of 1.1 granting 1.2. Since $b W \subseteq W$ is a homotopy equivalence, $H_{*}\left(W, b W ; Z^{t}\right)=0$. Thus 1.2 says $W$ has $e \leqq 1$ ends. If $e$ were $0, W$ would be compact and so $0=H_{n}\left(W, b W ; Z^{t}\right)=H^{0}(W ; Z) \cong Z$ by Poincaré duality [61, p. 357]. Hence $e=1$. ( $Z$ denotes the integers.)

Proof of 1.2. Coefficients for cohomology will be $Z$. For any $K \subset W$ we have an exact sequence for $(W, W-K)$

$$
0 \rightarrow Z=H^{0}(W) \rightarrow H^{0}(W-K) \rightarrow H^{1}(W, W-K) \rightarrow \cdots .
$$

Taking direct limit over $K$ compact in $W$ we get an exact sequence

$$
0 \rightarrow Z=H^{0}(W) \rightarrow H_{\infty}^{0}(W) \rightarrow H_{c}^{1}(W) \rightarrow \cdots .
$$

Then $\operatorname{rank}\left\{H_{c}^{1}(W)\right\}+1 \geqq \operatorname{rank} H_{\infty}^{0}(W)$.

Now $H_{c}^{1}(W) \cong H_{n-1}\left(W, b W ; Z^{t}\right)$ by Poincaré duality [52]. Hence the proof will be complete when we show that for all finite $k \leqq e, H_{\infty}^{\circ}(W) \supset Z^{k}$.

Since $W$ has $\geqq k$ ends, there exists a compact $K \subset W$ such that $W-K$ has at least $k$ unbounded connected components $L_{1}, L_{2}, \ldots, L_{k}, \ldots$

Then $H^{0}(W-K)=H^{0}\left(L_{1}\right) \oplus \cdots \oplus H^{0}\left(L_{k}\right) \oplus \cdots=Z^{k} \oplus \cdots$. The $Z^{k}$ survives in the direct limit $H_{\infty}^{0}(W)$. For, if $K^{\prime}$ is compact containing $K, H^{0}\left(L_{i}\right) \rightarrow H^{0}\left(L_{i}-K^{\prime}\right)$ is injective for each $i$ as $L_{i}$ is unbounded. Hence $H^{0}(W-K) \rightarrow H^{0}\left(W-K^{\prime}\right)$ injects the $Z^{k}$.

Definition. An inverse sequence of groups $A_{1} \leftarrow A_{2} \leftarrow A_{3} \leftarrow \cdots$ is essentially constant if there is a subsequence $B_{1}, B_{2}, B_{3}, \ldots$ of $A_{1}, A_{2}, A_{3}, \ldots$ such that composed homomorphisms

$$
B_{1} \stackrel{f_{1}}{\longleftarrow} B_{2} \stackrel{f_{2}}{\longleftarrow} B_{3} \stackrel{f_{3}}{\longleftarrow} \cdots
$$

induce isomorphisms of images

$$
\operatorname{Im}\left(f_{1}\right) \cong \operatorname{Im}\left(f_{2}\right) \stackrel{\cong}{\leftrightarrows} \operatorname{Im}\left(f_{3}\right) \cong \cdots .
$$

In this event, one clearly has $\operatorname{Im}\left(f_{i}\right) \cong \operatorname{proj} \lim _{j}\left\{A_{j}\right\}$.

Let $W$ be a topological manifold with one end. For each compact $K$ in $W$, let $K^{c}$ be the one unbounded component of $W-K$, and consider the inverse system of fundamental groups $\left\{\pi_{1}\left(K^{c}\right) \mid K\right.$ compact $\}$. We say that $\pi_{1}$ is essentially constant at $\infty$ if for a sequence $K_{1} \subset K_{2} \subset K_{3} \subset \cdots$ of compacta with $W=\bigcup$ int $K_{i}$ (where int $K_{i}$ is interior of $K_{i}$ ), the sequence induced by inclusions

$$
\mathscr{S}: \pi_{1}\left(K_{1}^{c}\right) \leftarrow \pi_{1}\left(K_{2}^{c}\right) \leftarrow \pi_{1}\left(K_{3}^{c}\right) \leftarrow \cdots
$$

is essentially constant. The choice of $\left\{K_{i}\right\}$, of base points, and of connecting base paths used to define $\mathscr{S}$ does not affect this property. Also $\pi_{1}(\infty)=\operatorname{proj} \lim (\mathscr{S})$ 
proves to be independent of these choices up to isomorphism in a preferred conjugacy class; and $\pi_{1}\left(K_{i}^{c}\right) \rightarrow \pi_{1}(W), i$ large, induces a homomorphism $\pi_{1}(\infty) \rightarrow$ $\pi_{1}(W)$ again determined within a conjugacy class. These elementary facts are verified in [40, Chapter III], [43].

We now state the main results.

THEOREM 1.3. Let $W^{w}, w \geqq 5$, be a connected manifold in DIFF or PL such that

(1) $\pi_{i}(W, b W)=0, i \leqq w-2$.

(2) $W$ has one end; $\pi_{1}$ is essentially constant at $\infty$ in $W$; and $\pi_{1}(\infty) \rightarrow \pi_{1}(W)$ is an isomorphism.

Then $W \cong b W \times[0,1)$.

REMARK 1.4. If $W \cong b W \times[0,1)$, (1) and (2) clearly hold, even when $b W$ is noncompact.

REMARK 1.5. Condition (2) can be verified without difficulty if $W$ is proper homotopy equivalent to $b W \times[0,1)$.

Combining this theorem with Proposition 1.1 one immediately gets

Open Collar TheOrem 1.6. Let $W^{w}, w \geqq 5$, be a connected manifold in DIFF or PL. Then $W \cong b W \times[0,1)$ if and only if

(i) $b W \subseteq W$ is a homotopy equivalence (so that $W$ has one end).

(ii) $\pi_{1}$ is essentially constant at $\infty$ and $\pi_{1}(\infty) \rightarrow \pi_{1}(W)$ is an isomorphism.

REMARK 1.7. A relative form of 1.6 follows directly from the one given. First consider the PL case. For any PL manifold $W$, a PL homeomorphism $f: M \cup\{b M$ $\times[0,1)\} \rightarrow b W$ can be extended to a PL homeomorphism $F: M \times[0,1) \rightarrow W$ if and only if $W \cong b W \times[0,1)$. (Here $M \cup b M \times[0,1)$ denotes a PL manifold with a collar attached to its boundary.) This follows immediately from the existence of a closed collar of $b M$ in $M$ and the isomorphism $[0,1) \times[0,1) \cong[0,1] \times[0,1)$.

In DIFF one must heed corners. One supposes $W^{w}$ is locally isomorphic to $R_{++}^{w}=\left\{\mathbf{x} \in R^{w} \mid x_{1} \geqq 0, x_{2} \geqq 0\right\}$ with corners along $f(b M)$. The statement in italics now applies to DIFF if $W \cong b W \times[0,1)$ is replaced (as it makes no sense) by $W_{s} \cong b\left(W_{s}\right) \times[0,1)$, where $W_{s}$ is $W$ with its corners rounded [7].

There remains in this section the task of proving Theorem 1.3. We propose to work simultaneously with DIFF and PL, but remark that in view of the CairnsHirsch theorem [13, Theorem 2.5], and Munkres [35, Theorem 1], the PL version implies the DIFF version-both in Theorem 1.3 and in the geometrical propositions below.

We will need some preliminaries. An admissible neighbourhood of $\infty$ in a manifold $W^{w}$ with boundary is a $w$-dimensional submanifold $V$ such that $V$ is closed as a subset, $W$-int $V$ is compact (int $V$ being the interior of $V$ as a subspace of $W$ ), and the pair $(W, V)$ is locally flat in the sense that it is locally isomorphic to $\left(R_{+}^{w}, R_{++}^{w}\right)$ where $R_{+}^{w}=\left\{\mathbf{x} \in R^{w} \mid x_{1} \geqq 0\right\}$ and $R_{++}^{w}=\left\{\mathbf{x} \in R^{w} \mid x_{1} \geqq 0, x_{2} \geqq 0\right\}$. One says that $V$ is a 0 -neighbourhood (of $\infty$ ) if $V$ and $b V$ are both connected. 
The first proposition is well known cf. [3, Lemma 6].

PROPOSITION 1.8. Let $W$ be a connected DIFF or PL manifold with connected boundary and one end. There exist arbitrarily small 0-neighbourhoods $V$ of $\infty$ in $W$.

Proof. Given a compact set $K$, one is required to produce a 0 -neighbourhood not meeting $K$. One starts with an admissible neighbourhood $V$ not meeting $K$ (from a proper Morse function in DIFF, or from a star of $K$ in PL). Neglecting compact components one makes $V$ connected. Finally, in order to make $v V$ connected one carves from $V 1$-handles joining the components of $b V$.

The next proposition requires some careful reasoning about fundamental groups.

Proposition 1.9. Suppose $W$ is a connected DIFF or PL manifold of dimension $\geqq 5$, having a connected boundary.

(A) If $W$ has one end and $\pi_{1}$ is essentially constant at $\infty$, then there exist arbitrarily small 0-neighbourhoods $V$ of $\infty$ such that $\pi_{1}(\infty) \rightarrow \pi_{1}(V)$ is an isomorphism.

(B) If in addition $\pi_{1}(\infty) \stackrel{\cong}{\rightarrow} \pi_{1}(W) \stackrel{\cong}{\longleftarrow} \pi_{1}(b W)$ then there exist arbitrarily small 0 -neighbourhoods $V$ such that $\pi_{1}(b V) \stackrel{\cong}{\rightarrow} \pi_{1}(V) \stackrel{\cong}{\rightarrow} \pi_{1}(W)$. Such $V$ are called 1neighbourhoods of $\infty$.

The proof is delayed until the end of this section in order to present:

Proof of Theorem 1.3. Stallings' engulfing techniques establish the required conclusion under the two hypotheses $\left(1^{\prime}\right)$ and $\left(2^{\prime}\right)$ below taken in place of (1) and (2) in the theorem:

$\left(1^{\prime}\right) \pi_{i}(W, b W)=0, i \leqq w-3$.

(2') There exist arbitrarily large compact sets $K$ such that $\pi_{i}(W, W-K)=0$, $i \leqq 2$.

We repeat the well-known proof for PL. In the DIFF case one triangulates, uses the PL case, then applies [15] or [35] to deduce a DIFF isomorphism.

Proposition. Let $W$ be a connected PL $n$-manifold, $n \geqq 5$ such that $\pi_{i}(W, b W)=0$, $i \leqq n-3$, and let $K \subset W$ be a compact set such that $\pi_{i}(W, W-K)=0, i \leqq 2$. If $U$ is a (collar) neighbourhood of $b W$, there exists a PL homeomorphism $h: W \rightarrow W$ fixing $b W$ such that $h(U) \supset K$.

The proof is explained by Stallings [47, §3], [46, §3.4], [48, §8.1].

Now let $W$ be a connected PL $n$-manifold $n \geqq 5$ such that $\left(1^{\prime}\right),\left(2^{\prime}\right)$ hold. Let $K_{1} \subset K_{2} \subset K_{3} \subset \ldots$ be a sequence of compacta in $W$ such that $\bigcup$ int $K_{1}=W$. Let $f_{0}: b W \times[0, \infty) \rightarrow W, f_{0}(x, 0)=x$, be any PL imbedding collaring $b W$. Suppose for an induction that the PL imbedding $f_{n}: b W \times[0, \infty) \rightarrow W, n \geqq 0$, has been defined so that $f_{n}(b W \times[0, n)) \supset K_{n}$. Find by $\left(2^{\prime}\right)$ a compact $L \subset W-f_{n}(b W \times[0, n))$ $=W_{n} \cong W$ such that $L \supset K_{n+1} \cap W_{n}$ and $\pi_{i}(W, W-L)=0, i \leqq 2$. The proposition provides a PL homeomorphism $h_{n}: W_{n} \rightarrow W_{n}$ fixing $b W_{n}$ such that $h_{n}\left(f_{n}[n, n+1]\right)$ $\supset L$. Define

$$
\begin{aligned}
f_{n+1} & =h_{n} f_{n} & & \text { on } b W \times[n, \infty) \\
& =f_{n} & & \text { on } b W \times[0, n]
\end{aligned}
$$


to complete the induction. Then one sets $f=f_{n}$ on $b W \times[0, n]$ for all $n$ getting a well defined PL homeomorphism

$$
f: b W \times[0, \infty) \rightarrow W
$$

as required.

Our task is to establish $\left(2^{\prime}\right)$. In fact

ASSERTION 1.10. If $V$ is any 1-neighbourhood of $\infty$ in $W$, (cf. Proposition 1.9), then $\pi_{i}(W, V)=0, i \leqq 2$.

REMARK 1.11. This is clear for $i=0,1$, but $\pi_{2}(W, V)=0$ depends via Poincaré duality on hypothesis (1) and the existence of small 1-neighbourhoods.

REMARK 1.12. To keep the algebra as simple as possible in proving the assertion, we use some elementary handlebody theory to be found in [32], [22] for DIFF or [51], [54], [63] for PL.

Now we prove the assertion. Let $K$ be the closure of $W-V$, a compact manifold, and let $X$ be $W$-int $V=K \cup b W$. Write $\partial_{+} K$ for $K \cap b V$ and $\partial_{-} K$ for $K \cap b W$. By first pushing $V$ away from $b W$ on any sufficiently large connected submanifold of $b W$, one arranges that $K, \partial_{-} K$, and $\partial_{+} K$ are all connected and $\partial_{+} K \subseteq K \partial_{2} \partial_{-} K$ give $\pi_{1}$-isomorphisms. We leave the reader to check that this is so with the help of the following lemma. (For example, in showing that $\partial_{-} K \subseteq K$ can give a $\pi_{1}$ isomorphism, $G \rightarrow H$ of the lemma will be $\pi_{1} b W \rightarrow \pi_{1}(b W \cup K)$.)

LEMMA 1.12. Let $G=\left\{x_{0}, x_{1}, x_{2}, \ldots ; r_{0}, r_{1}, r_{2}, \ldots\right\}$ and $H=\left\{x_{0}, y, x_{1}, x_{2}, \ldots\right.$; $\left.r_{0}, s, r_{1}, r_{2}, \ldots\right\}$ be groups with presentations where each of $x_{i}$ and $y$ is a set of generators while each of $r_{i}=r_{i}\left(x_{0}, x_{1}, \ldots, x_{i}\right)$ and $s=s\left(x_{0}, y\right)$ is a set of relators. Suppose the natural map $G \rightarrow H$ is an isomorphism and $y, s$ are finite sets. Then for all large $n$ the natural map

$$
G_{n}=\left\{x_{0}, \ldots, x_{n} ; r_{0}, \ldots, r_{n}\right\} \rightarrow H_{n}=\left\{x_{0}, y, x_{1}, \ldots, x_{n} ; r_{0}, s, r_{1}, \ldots, r_{n}\right\}
$$

is an isomorphism.

Proof of 1.12. Since $G \rightarrow H$ is onto and $y$ is finite, $y$ can be expressed as $y=\eta\left(x_{0}, x_{1}, \ldots, x_{a}\right)$ in $H$. Again because $y$ is finite $y=\eta\left(x_{0}, \ldots, x_{a}\right)$ is in $H_{n}$ for $n$ large, say $n>a^{\prime}(>a)$. Then

$$
H=\left\{x_{0}, x_{1}, \ldots ; r_{0}, s\left(x_{0}, \eta\left(x_{1}, \ldots, x_{a}\right)\right), r_{1}, \ldots\right\}
$$

and similarly for $H_{n}, n>a^{\prime}$. As $G \rightarrow H$ is injective, and $s$ is finite, $s\left(x, \eta\left(x_{0}, \ldots, x_{a}\right)\right)$ is, for the last presentation of $H$, in the consequence of $r_{0}, r_{1}, \ldots, r_{a^{\prime \prime}}$ for some $a^{\prime \prime}$. Then the same is true for $H_{n}$, for $n$ large, say $n>b\left(>\max \left(a^{\prime}, a^{\prime \prime}\right)\right)$. Thus $H_{n}=\left\{x_{0}, \ldots, x_{n} ; r_{0}, \ldots, r_{n}\right\}$ and $G_{n} \rightarrow H_{n}$ is an isomorphism for $n>b$.

Take a handle decomposition for $K$ on $\partial_{-} K$. Since $\pi_{i}\left(K, \partial_{+} K\right)=0, i \leqq 1$, easy cancellation theorems for (dual) 0 - and 1-handles tell us that no handles of dimension $(w-1)$ or $w$ need appear. 
By the rearrangement theorem for handlebodies, we can have the handles added in order of increasing dimension so that we obtain a handle decomposition of $X$ on $b W$

$$
X=b W \cup h^{0} \cup h^{1} \cup \ldots \cup h^{w-2}
$$

where $h^{i}$ is a finite thickened set of $i$-handles. Putting $X_{-1}=b W$ and $X_{i}=b W$ $\cup h^{0} \cup \ldots \cup h^{i}, i=0,1, \ldots, w-2$, we get a filtration

$$
X_{-1} \subset X_{0} \subset X_{1} \subset \cdots \subset X_{w-2}=X .
$$

Recall that $X_{i}$ has as a strong deformation retract $X_{i-1}$ with one $i$-cell attached for each handle. Let $\tilde{W}$ be the universal covering of $W$ and for $S \subset W$ let $\tilde{S} \subset \tilde{W}$ be the induced covering. Then the filtration $\tilde{X}_{-1} \subset \tilde{X}_{0} \subset \ldots \subset \tilde{X}$ gives a free complex $C_{*}(\tilde{X}, b \tilde{W})$, or $C_{*}$ for short, over $\Lambda=Z\left[\pi_{1} W\right]$ for the singular integral homology $H_{*}(\tilde{X}, b \tilde{W})$. By definition $C_{i}=H_{*}\left(\tilde{X}_{i}, \tilde{X}_{i-1}\right)$, which is a free module over $\Lambda$ with one generator for each $i$-handle; and $\partial: C_{i} \rightarrow C_{i-1}$ is the boundary for the triple $\left(X_{i}, X_{i-1}, X_{i-2}\right)$. We recall the well-known

Proposition 1.13. Let $X_{-1} \subset X_{0} \subset X_{1} \subset X_{2} \subset \cdots$ be a possibly infinite filtration of a space $X=\bigcup X_{i}$, homotopy equivalent to a C.W. complex. Suppose for each $i$ that $1 \mid X_{i-1}$ extends to a homotopy equivalence of $X_{i}$ with a space formed from $X_{i-1}$ by attaching a collection of cells by maps of the boundary spheres to $X_{i-1}$. Let a homotopy equivalence $f_{-1}: X_{-1} \rightarrow Y_{-1}$ to a $C . W$. complex be given. Then there exists a C.W. complex $Y \supset Y_{-1}$ with a filtration of $Y$ by subcomplexes $Y_{-1} \subset Y_{0}$ $\subset Y_{1} \subset \cdots$ such that $Y_{i}-Y_{i-1}$ consists of cells attached to $Y_{i-1}$, and there exists an extension of $f_{-1}$ to a homotopy equivalence $f: X \rightarrow Y$ such that $f \mid X_{i}$ gives a homotopy equivalence $X_{i} \rightarrow Y_{i}$.

The proof (not the statement) is contained in [30, $\$ 3.5,3.6,3.7,17.3]$.

Applying this proposition to $X \subset W$ we obtain a filtered complex $Y=Y_{w-2}$ $\supset \ldots \supset Y_{-1}$ and a filtration homotopy equivalence $f: X \rightarrow Y$. This is covered by a homotopy equivalence of (universal) coverings $\tilde{f}: \tilde{X} \rightarrow \tilde{Y}$ such that $\tilde{f} \mid \tilde{X}_{i}$ gives a homotopy equivalence $\tilde{X}_{i} \rightarrow \tilde{Y}_{i}$. It follows that $\tilde{f}$ induces a $\Lambda$-isomorphism of $C_{*}(\tilde{X}, b \tilde{W})$ with the similarly constructed complex $C_{*}\left(\tilde{Y}, \tilde{Y}_{-1}\right)$ (we identify the fundamental groups with $\pi_{1} W \subset \Lambda$ ).

Next form an infinite handle decomposition of $W$ on $X$ without handles of dimension $>(w-2)$. To accomplish this take a sequence of 1-neighbourhoods of $\infty, V_{0}=V \supset V_{1} \supset V_{2} \supset \cdots$ with $\bigcap V_{i}=\varnothing$ so that for each $i$ one obtains, as in the previous construction, a decomposition with no handles of dimension $>w-2$, for $V_{i-1}$-int $V_{i}$ on $b V_{i-1}\left({ }^{6}\right)$. The union of all these gives one of $W$ on $X$, viz. $W=X \cup h_{1} \cup h_{2} \cup h_{3} \cup \ldots$ where $h_{1}, h_{2}, \ldots$ are successively attached and suitably thickened handles.

(6) In DIFF one need not hesitate to round the corners at $b V_{t-1}$ in constructing the handle decomposition, for only the homotopy properties of the resulting filtration are needed. 
Applying Proposition 1.13 again we find a complex $Z \supset Y$ and a homotopy equivalence $g: W \rightarrow Z$ such that $g \mid X=f: X \rightarrow Y$ and $Z$ has one $k$-cell outside $Y$ for each $k$-handle added to $X$. Then $\tilde{f}: \tilde{X} \rightarrow \tilde{Y}$ extends to a homotopy equivalence $\tilde{g}: \tilde{W} \rightarrow \tilde{Z}$ covering $g$, where $\tilde{Z} \supset \tilde{Y}$ is a universal covering of $Z$. Let $C_{*}^{\prime} \equiv C_{*}\left(\tilde{Z}, \tilde{Y}_{-1}\right)$ be the $\Lambda$-complex from the filtration of $Z$ by skeletons. Then

$$
C_{*} \equiv C_{*}(\tilde{X}, b \tilde{W}) \cong C_{*}\left(\tilde{Y}, \tilde{Y}_{-1}\right) \subset C_{*}^{\prime}
$$

and $C_{*}^{\prime}$ is acyclic because

$$
H_{*}\left(C^{\prime}\right)=H_{*}\left(\tilde{Z}, \tilde{Y}_{-1}\right)=H_{*}(\tilde{W}, b \tilde{W})=0 .
$$

We now come to the heart of the proof. We have to show that $\pi_{2}(X, b V)=0$. But $\pi_{2}(X, b V) \cong H_{2}(\tilde{X}, b \tilde{V})$ and the complex $C_{*}(\tilde{X}, b \tilde{V})$ for $H_{*}(\tilde{X}, b \tilde{V})$ from the dual handle decomposition is naturally isomorphic (up to sign of the differentials) to

$$
\overline{\operatorname{Hom}}\left(C_{w-*}(\tilde{X}, b \tilde{V}), \Lambda\right) .
$$

This is Poincaré duality obtained by inspecting the geometry [33, pp. 395, 398]. By

$$
\overline{\operatorname{Hom}}(A, \Lambda) \text {, }
$$

or $\bar{A}$ for short, we always mean the left $\Lambda$-module of all additive homomorphisms $f: A \rightarrow \Lambda$ such that $f(g a)=f(a) \theta(g) g^{-1}$, for $g \in \pi_{1}(W) \subset \Lambda$, where $\theta: \pi_{1}(W) \rightarrow$ $\{ \pm 1\}$ is the orientation map. Equivalently $\bar{A}=\operatorname{Hom}_{\Lambda}(A, \Lambda)$ with the natural right action of $\Lambda$ suitably changed using $\theta$ to a left action.

Consider

$$
\bar{C}_{*}=\overline{\operatorname{Hom}}\left(C_{*}, \Lambda\right): \cdots \longleftarrow 0 \longleftarrow \bar{C}_{n-2} \stackrel{\bar{\partial}}{\longleftarrow} \bar{C}_{n-3} \stackrel{\bar{\partial}}{\longleftarrow} \bar{C}_{n-4} \stackrel{\bar{\jmath}}{\leftrightarrows} \cdots .
$$

We must establish that $\bar{C}_{n-2} \stackrel{\bar{\partial}}{\leftarrow} \bar{C}_{n-3}$ is onto, to show $H_{2}(\tilde{X}, b \tilde{V})=0$. Consider the commutative diagram

$$
\begin{aligned}
& 0 \longrightarrow C_{n-2} \stackrel{\partial}{\longrightarrow} C_{n-3} \stackrel{\partial}{\longrightarrow} \cdots \\
& \quad \stackrel{\downarrow}{\longrightarrow} C_{n-2}^{\prime} \stackrel{\partial^{\prime}}{\longrightarrow} C_{n-3}^{\prime} \stackrel{\partial^{\prime}}{\longrightarrow} \cdots .
\end{aligned}
$$

Since $C_{*}^{\prime}$ is acylic, it splits completely and there is a retraction $\rho^{\prime}: C_{n-2}^{\prime} \leftarrow C_{n-3}^{\prime}$ with $\rho^{\prime} \partial^{\prime}=1 \mid C_{n-2}^{\prime}$. Since $C_{n-2}^{\prime}=C_{n-2} \oplus R$ where $R$ is free with one basis element for each $(n-2)$-handle in $W$ outside $X$, we have a retraction $p: C_{n-2}^{\prime} \rightarrow C_{n-2}$. Let $\rho=\left(p \rho^{\prime}\right) \mid C_{n-3}$. Then $\rho \partial=1 \mid C_{n-2}$, and if we apply

\section{$\overline{\operatorname{Hom}}(, \Lambda)$,}

$\bar{\partial} \bar{\rho}=\overline{\rho \partial}=1 \mid \bar{C}_{n-2}$ proving $\bar{C}_{n-2} \stackrel{\bar{\partial}}{\leftarrow} \bar{C}_{n-3}$ is onto as desired. Hence $\pi_{2}(W, V)=0$, and the assertion about 1-neighbourhoods of $\infty$ is established. Modulo a proof of existence of small 1-neighbourhoods (Proposition 1.9) the proof of Theorem 1.3 is complete. 
Proof of Proposition 1.9. Part (A). Let $V_{1} \supset V_{2} \supset V_{3} \supset \cdots$ be a sequence of 0 neighbourhoods of $\infty$ with $V_{i+1} \subset$ int $V_{i}$ and $\cap V_{i}=\varnothing$, such that we have

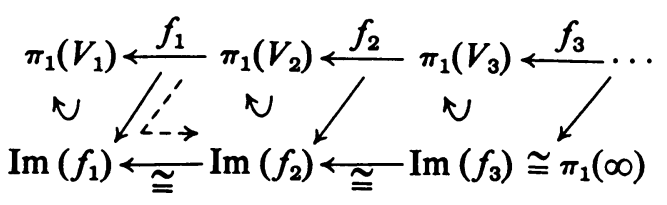

Writing $\pi_{1}\left(V_{3}\right)=\{x ; r\}$ in terms of generators and relators, one has $\pi_{1}\left(V_{2}\right)=\left\{x, x_{1}\right.$; $\left.r, r_{1}\right\}$, where $x_{1}$ is a finite set of generators and $r_{1}$ a finite set of relators. Write $\operatorname{Im}\left(f_{2}\right)=\{x ; r, s\}$ and let $\theta: \pi_{1}\left(V_{2}\right) \rightarrow \operatorname{Im}\left(f_{2}\right)$ be the retraction given by the dotted arrow.

Now we have a diagram

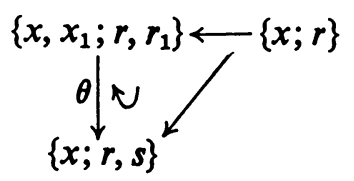

where $\theta$ is a retraction, and the other homomorphisms come from $x \mapsto x$. Then $\theta(x)=x$ and $\theta\left(x_{1}\right)=\xi_{1}(x)$ for some set $\xi_{1}$ of $\left|x_{1}\right|$ words in the generators $x$. We insert

LEMMA 1.14. In this situation $x \mapsto x$ gives an isomorphism

$$
\{x ; r, s\} \stackrel{\cong}{\longrightarrow}\left\{x, x_{1} ; r, r_{1}, x_{1}^{-1} \xi_{1}(x)\right\} \text {. }
$$

Proof of 1.14. By a "mapping cylinder" argument applied to $\theta$,

$$
\{x ; r, s\} \stackrel{\cong}{\longrightarrow}\left\{x, x_{1} ; r, r_{1}, x_{1}^{-1} \xi_{1}(x), s\right\} .
$$

As $\{x ; r, s\} \subset\left\{x, x_{1} ; r, r_{1}\right\}$ each element of $s$ can be written as a product of conjugates of $r, r_{1}$ by $x, x_{1}$. Thus the relators $s$ on the right of $\left(^{*}\right)$ are redundant.

Lemma 1.14 shows that the kernel of $f_{1}$ is the normal closure in $\pi_{1}\left(V_{2}\right)$ $=\left\{x, x_{1} ; r, r_{1}\right\}$ of the finite set of elements $x_{1}^{-1} \xi_{1}(x)$, and we know that $\pi_{1}(\infty)$ $\cong \operatorname{Im}\left(f_{1}\right)$. Let $y$ be one of the set $x_{1}^{-1} \xi_{1}(x)$. Since $\operatorname{dim} W \geqq 5$ we can represent $y$ by $b D \subset$ int $V_{2}$ where $b D$ is the boundary of an imbedded 2-disc $D \subset$ int $V_{1}$ that meets $b V_{2}$, transversely, in a finite number of circles in int $D$. By a familiar process of trading thickened innermost 2-discs in $D$ between $V_{2}$ and $W$-int $V_{2}$, [2, Lemma 3.1] we can change $V_{2}$ and $f_{1}$, killing the normal closure of $y$ and possibly more of kernel $\left(f_{1}\right)$. After one such process for each element of $x_{1} \xi_{1}^{-1}(x)$, we have a 0 neighbourhood $V \subset$ int $V_{1}$ such that this triangle commutes

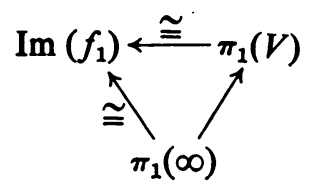


The map to $\pi_{1}(V)$ must be an isomorphism, so 1.9 part (A) is established.

Proof of Proposition 1.9. Part (B). Starting with a (small) 0-neighbourhood $U$ of $\infty$ provided by part (A) such that $\pi_{1}(\infty) \cong \pi_{1}(U) \cong \pi_{1}(W)$, we propose to carve from $U$ some 1- and 2-handles attached to $b U$ in $U$ so as to produce the required 1-neighbourhood.

Let $X=W$-int $U$, and consider the diagram of maps induced by inclusions

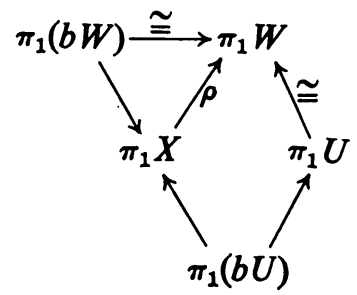

As $X=b U \cup\{$ compact manifold $\}$, if we write $\pi_{1}(b U)=\{x ; r\}$ we have $\pi_{1}(X)=$ $\left\{x, x_{1} ; r, r_{1}\right\}$ where $x_{1}$ is a finite set of generators and $r_{1}$ is a finite set of relators. Represent each element of $x_{1}$ as a loop in $X$ (with base point in $b U$ ). The diagram shows that each loop can be deformed inside $W$ to a loop in $U$. We imbed each deformed loop in $U$ so that the part in int $U$ gives a 1-handle in $U$ attached to $b U$. Subtracting these (thickened, open) 1-handles from $U$, we obtain a new 0-neighbourhood $U^{\prime}$ and a commutative diagram

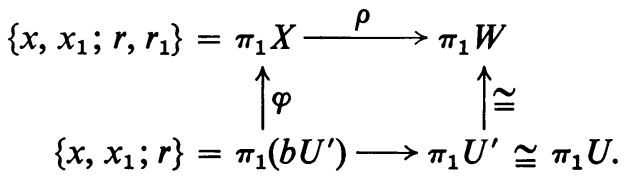

The free generators $x_{1}$ in $\pi_{1}\left(b U^{\prime}\right)$ correspond naturally to the 1-handles above. By definition, $\varphi$ is the quotient map of the presentations and does not arise from inclusions, although (by definition) the other maps do. However, $\varphi$ restricted to $\pi_{1}(b U)=\{x ; r\} \subset\left\{x, x_{1} ; r\right\}$ arises from $b U \subseteq X$. Hence commutativity is clear from our construction.

We can now show that the kernel of $\pi_{1}\left(b U^{\prime}\right) \rightarrow \pi_{1} U^{\prime} \cong \pi_{1}(\infty)$ is the normal closure of a finite set $\Sigma$. This kernel is equal to that of $\rho \varphi$. The kernel of $\varphi$ is the normal closure of $r_{1}=r_{1}\left(x, x_{1}\right)$. By Lemma 1.14 applied to the triangle in our first diagram, the retraction $\rho$ has as kernel the normal closure of a finite set $\left\{a_{1}, \ldots, a_{k}\right\}$. If $\varphi\left(a_{i}^{\prime}\right)=a_{i}$, then $\left\{a_{1}^{\prime}, \ldots, a_{k}^{\prime}\right\} \cup r_{1}$ is the required finite set $\Sigma$.

To complete the proof, find imbedded 2-discs in $U^{\prime}$, one for each element of $\Sigma$, so that each 2-disc $D$ meets $b U^{\prime}$ in $b D$ transversely, forming a loop that represents the corresponding element of $\Sigma$. We are now using the fact that $\operatorname{dim} W \geqq 5$. Thickening these discs and removing the resulting (open) 2-handles from $U^{\prime}$, one obtains the required 1-neighbourhood. This completes the proof of Proposition 1.9. The results of this section are now established. 
2. Detecting open regular neighbourhoods. Let $K$ be a closed connected subcomplex in a triangulation of a PL manifold $M$ (that may have a boundary). We seek homotopy criteria to decide whether a given open neighbourhood $W$ of $K$ in $M$ is PL homeomorphic fixing $K$ to the interior points of a regular neighbourhood $\left({ }^{7}\right)$ of $K$ in $M$. In this event one says that $W$ is an open regular neighbourhood of $K$ in $M$. Notice that $W$ need not be the interior of a (closed) regular neighbourhood of $K$ in $M$-e.g. $W$ may be $M$, or its closure may not be a manifold.

THEOREM 2.1. Suppose $\operatorname{dim} W \geqq \operatorname{dim} K+3$. Then the following (necessary) conditions on $W$ are, for $\operatorname{dim} W \geqq 5$, sufficient that $W$ be an open regular neighbourhood of $K$ in $M$.

(1) $W \cap b M$ is an open regular neighbourhood of $K \cap b M$ in $b M$.

(2) $K \subseteq W$ is a homotopy equivalence.

(3) $W$ has one end $\left({ }^{8}\right) ; \pi_{1}$ is essentially constant at $\infty$; and $\pi_{1}(\infty) \stackrel{\cong}{\longrightarrow} \pi_{1} W$.

Suppose next that $M$ is a DIFF manifold and suppose $K \subset M$ is a closed subset such that there exists a $C^{1}$-triangulation of $M, f: L \rightarrow M$ by a simplicial complex $L$ such that $f^{-1}(K)$ is a closed subcomplex. One says that an open neighbourhood $W$ of $K$ in $M$ is an open regular neighbourhood if $f^{-1}(W)$ is an open regular neighbourhood of $f^{-1}(K)$ in $L$ in the sense already defined $\left(^{9}\right)(L$ has a natural PL manifold structure). It is now a matter of definitions that Theorem 2.1 holds for DIFF if it holds for PL.

A most useful variation of 2.1 for DIFF is:

THEOREM 2.2. Let $W$ be a smooth manifold and $K \subset W$ a smooth, connected, properly imbedded, submanifold, such that $K$ meets $b W$ in $b K$, transversely. Suppose $\operatorname{dim} W \geqq \operatorname{dim} K+3$. Then, provided $\operatorname{dim} W \geqq 5$, the following three (necessary) conditions guarantee that $W$ admits the structure of a smooth vector bundle over $K$ :

(1) $b W$ can be made a smooth vector bundle over $b K$.

$\left({ }^{7}\right)$ For noncompact $K$, regular neighborhoods are treated in [39] and [55]. One can always take as regular neighborhood the closed star $S$ of $K$ in the second barycentric subdivision of a triangulation of $M$ having $K$ as subcomplex.

( ${ }^{8}$ Condition (2), $\pi_{0} K=0$, and $\operatorname{dim} W \geqq \operatorname{dim} K+2$ together imply that $W$ has just one end (see 1.1).

${ }^{(9)}$ If so, one would like to know (i) that for any other $C^{1}$ triangulation $g: L^{\prime} \rightarrow M$ such that $g^{-1}(K)$ is a subcomplex, $g^{-1}(W)$ is a PL open regular neighborhood of $g^{-1}(K)$. Also one wants (ii) uniqueness of these smooth open regular neighborhoods up to diffeomorphism fixing a neighborhood of $K$. For $\operatorname{dim} \geqq 5$ (and $\operatorname{dim} b M \geqq 5$ if $b M \neq \varnothing$ ) the proof is at hand: (i) follows easily from the Open Collar Theorem 1.6; (ii) follows from 1.6 and some smoothing theory of Munkres [35]. A proof valid in all dimensions can be based on B. Mazur's theory of canonical neighborhoods [25] applied to DIFF. But it is probably best to prove more--viz. establish simultaneously the notion of closed smooth regular neighborhood of $K$ in $M$ with the same uniqueness theorems as in PL. This is done by Hirsch in [14] for the compact case without boundary. Hirsch's arguments can now be adapted for the possibly noncompact case with boundary using [55]. 
(2) $K \subseteq W$ is a homotopy equivalence.

(3) $W$ has one end, $\pi_{1}$ is essentially constant at $\infty$ and $\pi_{1}(\infty) \rightarrow \pi_{1}(W)$ is an isomorphism.

Proof of Theorem 2.1. Let $R$ be a regular neighbourhood $\left({ }^{10}\right)$ of $K$ in $W, \partial R$ its frontier and int $R=R-\partial R$. We propose to show, using the relative form $\S 1.7(\mathrm{~b})$ of the Open Collar Theorem 1.6 that $W-$ int $R \cong b R \times[0,1)$. Then $W \cong$ int $R$ and 2.1 will be established.

Write $V$ for $W$-int $R$. Since $\operatorname{dim} K \leqq w-3$,

$$
\pi_{1} W \cong \pi_{1} V \cong \pi_{1}(\partial R) \cong \pi_{1} R \cong \pi_{1} K
$$

by inclusions. Then by excision in the universal coverings (that are indicated by a tilde)

$$
\pi_{*}(V, \partial R) \cong \pi_{*}(\tilde{V}, \partial \tilde{R}) \cong H_{*}(\tilde{V}, \partial \tilde{R})=0
$$

By $§ 1.2, V$ has one end and we have left to prove that $\pi_{1}$ is essentially constant at $\infty$ and $\pi_{1}(\infty) \cong \pi_{1} V$. We will use a heavy-handed argument that avoids geometric subtleties.

Since $R$ is a closed star neighbourhood of $K$ in a triangulation of $M$ with $K$ as a full subcomplex there exists a deformation fixing $\partial R, f_{t}, 0 \leqq t \leqq 1$, of $1 \mid(R-K)$ to a retraction onto $\partial R$, that has this special feature: For each compact $C \subset W$, there exists a compact $C^{\prime} \subset W$ such that $f_{t}(x) \in C$ implies $x \in C^{\prime}$. As $W$ has one end we can choose a sequence $U_{1} \supset U_{2} \supset U_{3} \supset \ldots$ of connected open sets with compact complements such that $\bigcap_{i} \bar{U}_{i}=\varnothing\left(\bar{U}_{i}=\right.$ closure of $\left.U_{i}\right)$. We can then find a subsequence (still called $U_{1}, U_{2}, U_{3}, \ldots$ ) such that for each $i, f_{t}, 0 \leqq t \leqq 1$, provides a deformation of $U_{i+1}-K \subseteq U_{i}-K$ to a map into $U_{i}$ int $R$ fixing $U_{i+1}-$ int $R$. Thus the diagram

$$
\begin{gathered}
U_{i}-\text { int } R \\
\curvearrowright U_{i+1}-\text { int } R \\
U_{i}-K \curvearrowright U_{i+1} \curvearrowright
\end{gathered}
$$

is homotopy commutative. As $U_{i+1}-K$ is connected

$$
\pi_{0}\left(U_{i} \text {-int } R\right) \leftarrow \pi_{0}\left(U_{i+1}-\text { int } R\right)
$$

is a constant map. Thus each set $U_{i}$-int $R$ has one unbounded component, say $U_{i}^{\prime}$ and $f_{1}$ gives maps $\pi_{1}\left(U_{i}^{\prime}\right) \leftarrow \pi_{1}\left(U_{i+1}-K\right)$ such that the sequence (with other maps from inclusions)

$$
\pi_{1}\left(U_{1}-K\right) \leftarrow \pi_{1} U_{1}^{\prime} \leftarrow \pi_{1}\left(U_{2}-K\right) \leftarrow \pi_{1} U_{2}^{\prime} \leftarrow \cdots
$$

factorizes the sequences for $\left\{U_{i}-K\right\}$ and $\left\{U_{i}^{\prime}\right\}$. Since $\operatorname{dim} K \leqq \operatorname{dim} W-3, \pi_{1}\left(U_{i}-K\right)$ $\cong \pi_{1} U_{i}$ by inclusion. As the sequence $\pi_{1} U_{1} \leftarrow \pi_{1} U_{2} \leftarrow \pi_{1} U_{3} \leftarrow \cdots$ is essentially

$\left({ }^{10}\right)$ This $R$ must not be confused with $R$ denoting the real line. 
constant we conclude from (*) that $\pi_{1} U_{1}^{\prime} \leftarrow \pi_{1} U_{2}^{\prime} \leftarrow \pi_{1} U_{3}^{\prime} \leftarrow \cdots$ is essentially constant and that we have a commutative diagram of isomorphisms

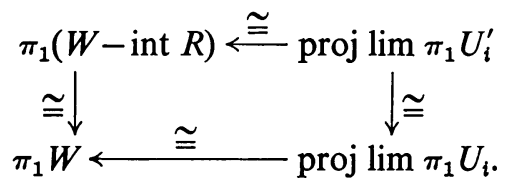

Condition (3) gives the bottom isomorphism; the top isomorphism is deduced and completes the proof of 2.1 .

Proof of 2.2. The proof of 2.1 can be applied to 2.2 with little modification. The last paragraph can even be abbreviated by convenient choice of $\left\{U_{i}\right\}$ and of $R$, which for the proof of 2.2 is a tubular neighbourhood of $K$ in $W$. Alternatively 2.1 implies 2.2 since an open tubular neighbourhood is precisely an open regular neighbourhood [14, Lemma 4].

TheOREM 2.3. In DIFF and in PL the following is true: Suppose $f:\left(M_{1}, b M_{1}\right) \rightarrow$ $\left(M_{2}, b M_{2}\right)$ is a (pair) homotopy equivalence of n-manifolds, such that $f^{*} \tau\left(M_{2}\right)$ and $\tau\left(M_{1}\right)$ are isomorphic after addition of trivial bundles. (Here $\tau\left(M_{i}\right)$ is the tangent bundle or microbundle in the given category.) Then for $s \geqq n+1$, there is an isomorphism

$$
F:\left(M_{1}, b M_{1}\right) \times R^{s} \rightarrow\left(M_{2}, b M_{2}\right) \times R^{s}
$$

(pair) homotopic to $f \times 1 \mid R^{s}$.

Proof of Theorem 2.3. Deform the map

$$
\left(M_{1}, b M_{1}\right) \stackrel{(f, 0)}{\longrightarrow}\left(M_{2}, b M_{2}\right) \times R^{s}
$$

to a proper map, then using $s \geqq n+1$ to a nearby proper imbedding $g$. Now $g$ has an imbedded normal bundle $\nu$ (for PL see Haefliger and Wall [11, Theorem 4]). By a familiar argument, the assumption about tangent bundles implies that $\nu$ is stably trivial. Since $s>n, \nu$ is actually trivial (for PL see [11, $\S 3$, Corollary]). Then the total space $E(\nu) \cong g\left(M_{1}\right) \times R^{s}$ and $g$ extends to the required isomorphism $F: M_{1} \times R^{s} \rightarrow M_{2} \times R^{s}$. For DIFF use 2.2 here, and for PL use 2.1 (or its proof).

REMARKS on 2.3. (a) For $M_{i}$ compact (and closed) the above result is due to Mazur [23], [65]. Hirsch extended Milnor's proof [28] to the case where $f$ is a proper homotopy equivalence [16]. In 1968 Kuiper and Burghelia [60] have established 2.8 for separable smooth Hilbert manifolds of dimension $\infty$, by using Milnor's proof and a result of Bessaga peculiar to dimension $\infty$. Thus far, all efforts to prove 2.3 using the elementary ideas of Mazur or Milnor have only led to fallacies (see $\$ 0)$.

(b) Theorem 2.3 with $s \gg n$ is true for TOP (cf. [26], [41]). In fact it follows from 2.3 for DIFF by an argument of Hirsch given in [16, \$4]. The DIFF manifold to be associated to $M_{i}, i=1,2$, is the total space of a normal microbundle $\nu$ for an imbedding of $\left(M_{i}, b M_{i}\right)$ in $\left(D^{t}, b D^{t}\right), t$ large, chosen by the Kister-Mazur theorem 
[16] to be an $R^{t-n}$-bundle. The restriction $\nu \mid b M_{i}$ is to be a normal bundle for the imbedding of $b M_{i}$ in $b D^{t}$. The usual existence theorem for normal microbundles [31] applies only to manifolds without boundary. Existence of $\nu$ follows with the help of Hirsch [17, Theorem 1(c)].

Theorem 2.3 is not definitive even for unbounded manifolds. Let $f: M_{1} \rightarrow M_{2}$ be a homotopy equivalence of unbounded $n$-manifolds. We place ourselves consistently in DIFF or in PL. Suppose $f$ is tangential-i.e. $f^{*} \tau\left(M_{2}\right)$ is stably isomorphic to $\tau\left(M_{1}\right)$. Suppose in addition that $M_{1}, M_{2}$ each admit a possibly infinite handle decomposition with handles of dimension $\leqq k<n / 2$ only $\left({ }^{11}\right)$. J. Milnor suggested the

QUESTION 2.4. Is $f$ homotopic to an isomorphism?

If $f$ is proper and one demands a proper homotopy to an isomorphism, the answer is no in general. Examples occur for $M_{1}=M_{2}=S^{2} \times R^{r}, r \geqq 5$, because the $J$-homomorphism

$$
0=\pi_{2} O(r) \rightarrow \pi_{2} G(r)=\pi_{r+1}\left(S^{r-1}\right)=Z_{2}
$$

is not onto. ( $G(r)$ is the space of degree \pm 1 maps $S^{r-1} \rightarrow S^{r-1}$.)

When $M_{i}, i=1,2$, is the total space of a $R^{k+1}$-bundle over a $k$-manifold $N_{i}^{k}$, the proof of 2.3 gives a positive answer. For a positive answer in dimensions $\geqq 5$, see [41].

For amusement we unknot a whole forest of $R^{k}$ 's in $R^{n}, n-k \geqq 3$.

THEOREM 2.5. Suppose $N$ is a union of $s$ disjoint euclidean spaces $R^{k_{1}}, R^{k_{2}}, \ldots$, $R^{k_{s}}, k_{i} \leqq n-3$, smoothly and properly imbedded in $R^{n}, n \geqq 5$. Then $\left(R^{n}, N\right)$ is diffeomorphic to the standard pair consisting of the cosets $R^{k_{i}}+(0, \ldots, 0, i) \subset R^{n}, i=1$, $\ldots, s$.

Proof of 2.5. There exists a smoothly, properly embedded copy of $R^{1}$ that meets each component of $N$ in a single point, transversely. As any two smooth proper embeddings of $R^{1}$ into $R^{n}$ are related by a diffeomorphism (use 2.2 or [4]), we can arrange that the $i$ th component of $N$ meets the last coordinate axis in $(0, \ldots, 0, i)$, transversely. Then using uniqueness up to ambient isotopy of tubular neighbourhoods of the last axis [29], [60], one can further arrange that $N$ coincides with the standard cosets near this axis. One verifies next that one open regular neighbourhood $W$ of $N \cup$ \{last axis\} gives a pair ( $W, N \cup$ \{last axis $\}$ ) diffeomorphic to the standard pair with the last axis added. Finally apply 2.1 to complete the proof.

REMARKS. The above result is taken from [40]. Using the fact that $R^{n-1}$ unknots in $R^{n}, n \neq 4$, [47], one can replace $k_{i} \leqq n-3$ in 2.5 by $k_{i} \neq n-2$. A PL version of 2.5 can be deduced by smoothing [13].

${ }^{(11}$ It is equivalent, at least for $n \geqq 5$ (where 2.1 works), to suppose that $M_{i}$ is an open regular neighbourhood of a closed $k$-subcomplex, $i=1,2$. 
We conclude this section with another amusing result that was suggested by D. B. A. Epstein and was proved with him. There is a well-known

Proposition 2.6. Suppose $X$ is a PL (or TOP) oriented closed $n$-manifold, $n \geqq 5$. If there is a degree 1 map $S^{n} \rightarrow X$, then $X \cong S^{n}$.

The degree 1 map shows that $X$ is a homotopy sphere and one applies the Poincaré Conjecture proved for $n \geqq 5$ [38] (see 2.9, 2.10, 2.11 below).

Epstein suggested the corresponding characterization of $R^{n}, n \geqq 5$ :

TheOREM 2.7. Suppose $X$ is a DIFF or PL oriented unbounded $n$-manifold, $n \geqq 5$. If there is a proper degree 1 map $R^{n} \rightarrow X$, then $X \cong R^{n}$.

Its proof also establishes:

Theorem 2.8. Suppose $X$ is a DIFF or PL oriented n-manifold, $n \geqq 5$ with nonempty simply connected boundary $b X$. If there is a proper degree 1 map

$$
f: b X \times[0,1) \rightarrow X
$$

with $f \mid b X \times 0$ the inclusion, then

$$
X \cong b X \times[0,1) .
$$

Of course the proofs are based on the Open Collar Theorem 1.6. It is the homotopy theoretic form of the $\pi_{1}$ condition at $\infty$ that makes the proofs painless. We present three lemmas which clearly establish $2.7,2.8$ in view of 1.6 and 2.1, 2.2.

Suppose $M$ and $X$ are oriented connected topological $n$-manifolds and.

$$
f:(M, b M) \rightarrow(X, b X)
$$

is a proper map of degree 1 . Let $[M],[X]$ be the preferred free generators of $H_{c}^{n}(M, b M), H_{c}^{n}(X, b X)$, each Poincaré dual to a point. ( $H_{c}^{*}$ is integral singular cohomology based on cochains with compact support.) Then the degree $\operatorname{deg}(f)$ is defined by

$$
f^{*}[X]=\operatorname{deg}(f)[M] .
$$

LEMMA 2.9. $f_{*}: \pi_{1} M \rightarrow \pi_{1} X$ is surjective.

LEMMA 2.10. If $M$ has one end, $\pi_{1}$ is essentially constant at $\infty$ in $M$ and $\pi_{1}(\infty)=1$, then the same is true in $X$.

LeMma 2.11. $f_{*}: H_{*}(M, b M) \rightarrow H_{*}(X, b X)$ is a retraction of groups.

Proof of 2.9. Let $\hat{X} \stackrel{p}{\rightarrow} X$ be the covering corresponding to $f_{*} \pi_{1} M \subset \pi_{1} X$. Then $f$ factors through $\hat{X}$ by a necessarily proper map $\hat{f}: M \rightarrow \hat{X}$, and one knows that $\operatorname{deg}(f)=\operatorname{deg}(p \hat{f})=\operatorname{deg}(p) \operatorname{deg}(\hat{f})$. Hence $\operatorname{deg}(p)= \pm 1, \hat{X}=X[9, \S 2]$, and so $f_{*} \pi_{1} M=\pi_{1} X$ as required. 
Proof of 2.10. If $X$ had $>1$ end, $M$ clearly would too. So we can find $V_{1} \supset V_{2}$ $\supset V_{3} \supset \cdots$ connected neighbourhoods of $\infty$ in $X$ with $\cap V_{i}=\varnothing$. Let $U_{i}$ be the unbounded component of $f^{-1}\left(V_{i}\right)$ and consider the ladder given by $f$

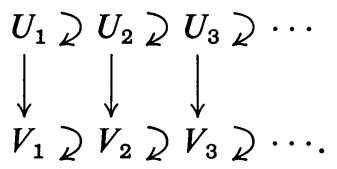

As $\cap \bar{U}_{i}=\varnothing$, we can arrange by picking a subsequence that $\pi_{1} U_{i+1} \rightarrow \pi_{1} U_{i}$ has image 1 . Certainly $f \mid U_{i}: U_{i} \rightarrow V_{i}$ is proper. It has degree 1 for we can deform $f$ slightly to $f^{\prime}$ on a compactum in $U_{i}$ so that $f^{\prime}$ is transverse at a point $p$ in $V_{i}$ chosen so near $\infty$ that $f^{-1}(p) \subset U_{i}$. Then the geometrical interpretation of degree shows that $\operatorname{deg}\left(f^{\prime} \mid U_{i}\right)=\operatorname{deg}\left(f^{\prime}\right)$ whence $\operatorname{deg}\left(f \mid U_{i}\right)=\operatorname{deg}(f)=1$.

See [9, §2.1b, §4.2]. Now 2.9 implies $\pi_{1} V_{i+1} \rightarrow \pi_{1} V_{i}$ has image 1. (REMARK. Here is the one point where $\pi_{1} b X=1$ is really essential in the proof of 2.8.)

Proof of 2.11. Left inverse to $f_{*}$ is (P.D.) $f^{*}$ (P.D.) $)^{-1}$ in the diagram

$$
\begin{array}{cc}
H_{c}^{i}(M) \stackrel{f^{*}}{\longleftarrow} & H_{c}^{i}(X) \\
\text { P.D. } \downarrow & \cong \downarrow \text { P.D. } \\
H_{n-i}(M, b M) \stackrel{f_{*}}{\longrightarrow} & H_{n-i}(X, b X)
\end{array}
$$

where P.D. denotes Poincaré duality. Commutativity of $\left(^{*}\right)$ wants checking if the manifolds are not compact. For any compact $K \subset X$ and compact $L \supset f^{-1} K$ one has

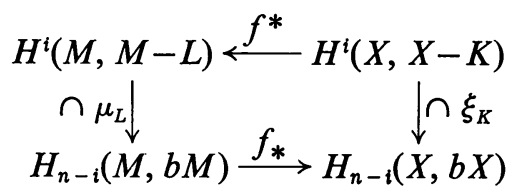

where $\xi_{K} \in H_{n}(X,(X-K) \cup b X)$ is the orientation class as is $\mu_{L} \in H_{n}(M,(M-K)$ $\cup b M)$, and the downward maps are cap products with these classes. Now $f_{*} \mu_{L}$ $=\xi_{K}$, by the geometrical interpretation of degree $\mathrm{cf}$. $[9, \S 2.1 \mathrm{~b}, \S 4.3]$. So $(\S)$ commutes by a cap product rule. Finally, $(\dagger)$ is the direct limit of such squares $(\S)$; so 2.11 is established.

3. Applications to group actions. Here we prove Theorem 3.1 stated in the introduction and compare the situation for actions on closed disc bundles.

There is given for 3.1 a DIFF manifold $W$ without boundary, which can be made the total space of a smooth vector bundle over a given manifold $M \subset W$. A smooth action $\alpha: G \times W \rightarrow W$ of a compact Lie group $G$ on $W$ is also given such that $M$ is the set of points fixed by $\alpha$, and for $x \in W-M$ the isotropy subgroups $G_{x} \subset G$ are all conjugate-i.e. $\alpha$ has just one orbit type on $W-M$.

THEOREM 3.1. If $\operatorname{dim} W-\operatorname{dim}\left(G / G_{x}\right) \geqq 5, W$ admits a structure $\zeta$ of euclidean vector bundle over $M$ so that $\alpha$ respects fibers of $\zeta$ and gives isometries of each fiber. 
We will state a relative version in $\$ 3.3$ below.

Proof of Theorem 3.1. In outline we follow the argument of [4, Theorem B]. Certainly we can assume for the proof that $W$ is connected. A Riemannian metric on $W$ averaged with respect to the action can be used to construct an open $\alpha$ invariant tubular neighbourhood $E$ of $M$ which is a euclidean vector bundle over $M$ on which $\alpha$ respects fibers and acts orthogonally. $E$ is called an open $\alpha$-equivariant tube and the unit disc bundle $E_{1} \subset E$ is called a closed $\alpha$-equivariant tube (for $M$ in. $W$ ). Write $V=W$-int $E_{1}, V_{0}=E-$ int $E_{1}, B=V^{\alpha}$ (the orbit space of $\alpha$ on $V$ ) and $B_{0}=V_{0}^{\alpha}$. Since $\alpha$ has just one orbit type on $V, B$ is a manifold with boundary $b B=b V^{\alpha}$, and $V \rightarrow V^{\alpha}=B$ is a smooth bundle with fiber $G / G_{x}$ [38]. Uniqueness up to ambient isotopy of (ordinary) tubular neighbourhoods of $M$ shows that $V \cong b V \times[0,1)$, which suggests:

AsSERTION 3.2. $\mathrm{B} \cong b B \times[0,1)$.

The proof uses the Open Collar Theorem 1.6, and is postponed. Since $\alpha$ is orthogonal on $E, B_{0} \subset B$ is a collar neighbourhood of $b B$. Hence the usual theorem for uniqueness of collar neighbourhoods up to ambient isotopy implies that there exists a smooth isotopy $f_{t}, 0 \leqq t \leqq 1$, of $B_{0} \subseteq B$ through smooth imbeddings, that fixes a neighbourhood in $B_{0}$ of $b B_{0}=b B$ and terminates at an isomorphism $f_{1}$ of $B_{0}$ onto $B$.

Consider the smooth bundle $V \rightarrow V^{\alpha}=B$ and its restriction $V_{0} \rightarrow V_{0}^{\alpha}=B_{0}$. Write $H$ for the isotropy subgroup $G_{x_{0}}$ at a fixed point $x_{0} \in B_{0}$. If $N$ is the normalizer of $H$ in $G$, write $\Gamma=N / H$. Then $V \rightarrow B$ has fiber $G / H$ and group $\Gamma$ acting on $G / H$ on the right. Let $P(V)=\left\{x \in V \mid G_{x}=H\right\}, P\left(V_{0}\right)=\left\{x \in V_{0} \mid G_{x}=H\right\}$. Then $P(V) \rightarrow B$ is a principal $\Gamma$-bundle with $\Gamma$ acting on $P(V)$ on the left. The vital fact for us is that the associated bundle

$$
(G / H)_{\Gamma}^{\times} P(V) \rightarrow B,
$$

with the obvious action of $G$ on the left, is naturally isomorphic to $V \rightarrow B$ with the action $\alpha$ of $G$. See [21, §1.1] and [50].

Apply the bundle covering homotopy theorem of Steenrod [49, §11.3] (in its differentiable version) to the homotopy $f_{t}, 0 \leqq t \leqq 1$, of $B_{0} \subseteq B$.

One obtains a smooth bundle map $\varphi$ of $P\left(V_{0}\right)$ onto $P(V)$ which covers $f_{1}: B_{0} \cong B$ and is the identity over a neighbourhood of $b B_{0}=b B$. The observation of the previous paragraph converts $\varphi$ into an isomorphism $\bar{\varphi}$ of $V_{0}$ onto $V$ fixing a neighbourhood of $b V_{0}=b V$ and respecting the action of $G$. Extending $\bar{\varphi}$ by the identity on $E_{1} \subset V$ one has an equivariant isomorphism of $E=E_{1} \cup V_{0}$ onto $W=E_{1} \cup V$, which establishes Theorem 3.1.

We return to

Proof of Assertion 3.2. As $b V \subseteq V$ is a homotopy equivalence the fibration $G / H \rightarrow V \rightarrow B$ tells us that $b B \subseteq B$ is a homotopy equivalence. As $W$ is (assumed) connected, either $b V$ is connected, or $W=M \times R^{1}$. In either case $b B$ must be connected. Hence $B$ has one end by $\S 1.1$. Since $\operatorname{dim} B=\operatorname{dim} V-\operatorname{dim}(G / H) \geqq 5$, 
the Open Collar Theorem 1.6 will yield $B \cong b B \times[0,1)$ provided we can show that $\pi_{1}$ is stable at $\infty$ in $B$ and $\pi_{1}(\infty) \cong \pi_{1}(B)$.

Choose a sequence $B_{1} \supset B_{2} \supset B_{3} \supset \ldots$ of connected neighbourhoods of $\infty$ in $B$ such that $\cap \bar{B}_{i}=\varnothing$. We aim to show that the sequence

$$
\pi_{1}(B) \leftarrow \pi_{1}\left(B_{1}\right) \leftarrow \pi_{1}\left(B_{2}\right) \leftarrow \cdots
$$

induces isomorphisms

$$
\pi_{1}(B) \stackrel{\cong}{\leftrightarrows} \operatorname{Im} \pi_{1}\left(B_{1}\right) \stackrel{\cong}{\leftrightarrows} \operatorname{Im} \pi_{1}\left(B_{2}\right) \stackrel{\cong}{\leftrightarrows}
$$

at least after $\left\{B_{i}\right\}$ is replaced by a subsequence.

Since the bundle $V \rightarrow B$ has compact fiber, the sets $V_{i}=q^{-1} B_{i}, i=1,2,3, \ldots$ are neighbourhoods of infinity in $V$; and clearly $\cap \bar{V}_{i}=\varnothing$. We know that $V$ has one end (\$1.1) unless $W=M \times R^{1}$. In either case $\pi_{0}\left(V_{1}\right) \leftarrow \pi_{0}\left(V_{2}\right) \leftarrow \cdots$ is essentially constant and we are able to choose base points and connecting base paths so that $\pi_{1}\left(V_{1}\right) \leftarrow \pi_{1}\left(V_{2}\right) \leftarrow \cdots$ is well defined and essentially constant. Hence we can (and do) replace $\left\{B_{i}\right\}$ by a subsequence so that isomorphisms

$$
\pi_{i}(V) \stackrel{\cong}{ } \operatorname{Im}\left(\pi_{i} V_{1}\right) \stackrel{\cong}{ } \operatorname{Im}\left(\pi_{i} V_{2}\right) \stackrel{\ldots}{\leftrightarrows}
$$

are induced for $i=0,1$.

From the rows of fibrations (where $F$ stands for $G / H$ ):

$$
\begin{aligned}
& \begin{array}{ccc}
\vdots & \vdots & \vdots \\
11 & \curvearrowright & \curvearrowright
\end{array} \\
& F \rightarrow V_{2} \quad B_{2} \\
& \| \curvearrowright \curvearrowright \\
& F \rightarrow V_{1} \rightarrow B_{1} \\
& \| \curvearrowright \curvearrowright \\
& F \rightarrow V \rightarrow B
\end{aligned}
$$

we deduce exact rows in

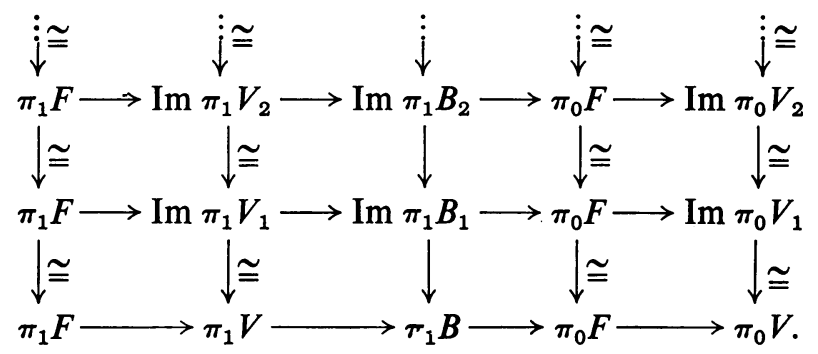

We established the second and last columns of isomorphisms in the last paragraph. A five-lemma argument now shows that the middle column consists of isomorphisms. This establishes Assertion 3.2 and completes the proof of Theorem 3.1. 
3.3. Relative version of Theorem 3.1. There is a relative version of 3.1 in which $M$ and $W$ have boundaries and $\alpha$ is supposed orthogonal for some euclidean vector bundle structure $\xi$ for $b W$ over $b M$. The statement of 3.1 again holds true, and is proved much as before with the help of $\$ 1.7(b)$. Further, using a theorem of uniqueness up to equivariant ambient isotopy for equivariant tubes $\left({ }^{12}\right)$, one shows that the bundle structure $\zeta$ promised by 3.1 can be required to coincide with $\xi$ over $b M$.

We conclude $\S 3$ with a discussion of the situation for actions on closed disc bundles. For Propositions 3.4 and 3.5 below we suppose that $W$ admits a structure of smooth closed disc bundle with orthogonal group over an unbounded submanifold $M \subset W . \alpha$ is still a smooth action of a compact Lie group $G$ on $W$ with fixed point set $M$ and one orbit type outside $M$. Let $H$ be $G_{x_{0}}$ for $x_{0}$ a point of $W-M$.

Proposition 3.4. In the above situation suppose $G$ is connected, $\operatorname{dim} W-$ $\operatorname{dim}(G / H) \geqq 6$ and $M$ is compact, simply connected. Then $\alpha$ acts orthogonally with respect to a suitable orthogonal disc bundle structure for $W$ over $M$.

Proof of 3.4. From the information in [ $3, \S 1$, Remark], one deduces that the orbit space $b W^{\alpha}$ must be simply connected. Then one employs the method of proof 3.1, using the $h$-cobordism Theorem [32] in place of the Open Collar Theorem 1.6 (cf. $[50, \S 3])$.

To indicate that the general case is more complicated, we offer a classification theorem in a second special case.

Consider smooth actions $\beta: G \times W \rightarrow W$ that fix $M$ pointwise, give the same action as $\alpha$ on the normal bundle $\nu(M)$ of $M$ in $W$, and have just one orbit type outside $M$. Let $\alpha_{0}$ be the common action on $\nu(M)$. For two such actions $\beta_{1}, \beta_{2}$ we say $\beta_{1} \sim \beta_{2}$ if there exists a smooth automorphism $F: W \rightarrow W$ that takes $\beta_{1}$ to $\beta_{2}$, that fixes $M \subset W$ and that gives the identity automorphism of $\nu(M)$. Let $\mathscr{A}=\mathscr{A}\left(\alpha_{0}\right)$ be the collection of resulting equivalence classes of actions.

Proposition 3.5. In the above situation, suppose $G$ is finite, $M$ is compact connected without boundary, and $\operatorname{dim} W \geqq 6$. Then $\mathscr{A}$ is in natural 1-1 correspondence with the kernel of the transfer homomorphism $t: W h \pi_{1}\left(b W^{\alpha}\right) \rightarrow W h \pi_{1}(b W)$ of Sylvia de Christ [6] for the subgroup $\pi_{1}(b W) \subset \pi_{1}\left(b W^{\alpha}\right)$. This subgroup is normal and of finite index.

Remark 3.6: Relativizing 3.4 AND 3.5. Proposition 3.4 continues to hold if $M$ is allowed a boundary and one supposes that $W$ is diffeomorphic (fixing $M$ ) to a smooth disc bundle over $M$ with its corners smoothed. With this change 3.5 continues to hold provided $b W$ is replaced in the statement by $b(W-M)$. The proofs require the relative form of the $h$ - or $s$-cobordism theorem [22], but use no new ideas.

(12) See [62]. 
Before proving 3.5 we explain the transfer. Let $A$ be a discrete group and $A_{0} \subset A$ a subgroup such that $A=A_{0} a_{1} \cup \cdots \cup A_{0} a_{d}$, $d$ finite. Then the integral group ring $Z[A]$ is a free module over $Z\left[A_{0}\right] \subset Z[A]$ with $d$ free generators $a_{1}, \ldots, a_{d}$. (Modules will be left modules.) In this way each $Z[A]$-automorphism of $Z[A]^{n}$, $n$ finite, becomes a $Z\left[A_{0}\right]$-automorphism of $Z\left[A_{0}\right]^{d n}$. There results a transfer homomorphism $t: \mathrm{Wh}(A) \rightarrow \mathrm{Wh}\left(A_{0}\right)$. Sylvia de Christ [6] observed that if $c=\left(W ; V, V^{\prime}\right)$ is a smooth compact $h$-cobordism and $\bar{c}=\left(\bar{W} ; \bar{V}, \bar{V}^{\prime}\right)$ is a covering with finitely many leaves, then $\bar{c}$ is an $h$-cobordism with torsion $\tau(\bar{c})=t(\tau(c))$; where $t$ is the transfer

$$
t: \mathrm{Wh}\left(\pi_{1} V\right) \rightarrow \mathrm{Wh}\left(\pi_{1} \bar{V}\right) .
$$

The transfer is not easy to calculate, but for $A_{0}$ normal in $A$ there is a helpful identity. Let $A_{0} \subseteq A$ induce $i_{*}: \mathrm{Wh}\left(A_{0}\right) \rightarrow \mathrm{Wh}(A)$. For any $a \in A$ let $x \mapsto x^{a}$ be the automorphism of Wh $\left(A_{0}\right)$ induced by the automorphism $g \mapsto a g a^{-1}$ of $A_{0}$. The identity is

$$
t\left(i_{*}(x)\right)=x^{a_{1}}+\cdots+x^{a_{d}}, \quad x \in \mathrm{Wh}\left(A_{0}\right)
$$

and can be verified by a matrix calculation. The same identity occurs for homology of groups. If there is a right inverse $j: A \mid A_{0} \rightarrow A$ of $A \rightarrow A / A_{0}$, one can check that $t j_{*}(y)=0$ for $y \in \mathrm{Wh}\left(A / A_{0}\right)$.

Proof of Proposition 3.5 (in outline). Assuming $b W$ is connected, the projection $b W \rightarrow b W^{\alpha}$ to the orbit space is a connected covering. Hence the isotropy subgroup is the same normal subgroup $H$ throughout $b W$. Thus $b W \rightarrow b W^{\alpha}$ has $|G / H|$ leaves and as many covering transformations, which shows $\pi_{1}(b W) \subset \pi_{1}\left(b W^{\alpha}\right)$ is a normal subgroup. If $b W$ is not connected, $W \cong M \times[-1,1]$ and one sees that $\alpha$ must identify the components of $b W$, whence $\pi_{1}(b W)=\pi_{1}\left(b W^{\alpha}\right)=\pi_{1} M$.

If $\beta: G \times W \rightarrow W$ represents an element of $\mathscr{A}$, let $T$ be a $\beta$-equivariant tube for $M$ in int $W$. Let $V=W$-int $T$ and consider the cobordism $c=\left(V^{\beta} ; b T^{\beta}, b W^{\beta}\right)$. It is an $h$-cobordism and its torsion $\tau(c) \in \mathrm{Wh}\left(\pi_{1}\left(b W^{\beta}\right)\right)$ satisfies $t \tau(c)=0$ as the covering. $(V ; b T, b W)$ is a product cobordism. Since $\alpha$ and $\beta$ give the same action $\alpha_{0}$ on $\nu(M)$, there is a natural homotopy equivalence of the map $b W \rightarrow b W^{\beta}$ with $b W \rightarrow b W^{\alpha}$. Hence $\tau(c)$ is naturally an element of the kernel of $t: \mathrm{Wh} \pi_{1}\left(b W^{\alpha}\right)$ $\rightarrow \mathrm{Wh} \pi_{1}(b W)$. We let $\beta \rightarrow \tau(c)$ define $\theta: \mathscr{A} \rightarrow \operatorname{ker}(t)$.

That $\theta$ is well defined follows from the uniqueness theorem for equivariant tubes. (See footnote 12.) Injectivity of $\theta$ follows easily from the $s$-cobordism theorem [22, §11.3], [33]. Surjectivity of $\theta$ follows from Stallings' important observation $[22, \S 11.1]$ that for a prescribed closed smooth manifold $N$, and prescribed $x \in \mathrm{Wh}\left(\pi_{1} N\right)$ there exists an $h$-cobordism with left end $N$ and torsion $x$.

This completes the proof of 3.5 .

Discussion of 3.5. Suppose $\alpha$ is orthogonal. Every $x \in \mathscr{A}$ which under the bijection $\mathscr{A} \leftrightarrow \operatorname{kernel}(t)$ gives an element in the subgroup $H \subset \operatorname{kernel}(t)$ of elements of the form $y+(-1)^{w-1} y^{*}(w=\operatorname{dim} W, *$ is the duality involution of Milnor 
[22, p. 398]) has a representative $\alpha^{\prime}$ which coincides with $\alpha$ on $b W$. This is because $\alpha^{\prime}$ can be constructed (see proof of 3.5) using a composition of an $h$-cobordism $\left(A ; B, B^{\prime}\right)$ with its reverse $\left(A ; B^{\prime}, B\right)[22$, p. 394]. These actions $H \subset \mathscr{A}$ have the following interest once mentioned to us by Wu-Yi Hsiang. Suppose in the situation of 3.5 that $W$ is a tubular neighbourhood of $M$ in a larger manifold $X$ and that the given (orthogonal) action $\alpha$ is the restriction to $W$ of a given action $\beta$ on $X$. Then one can alter $\beta$ by changing $\alpha$ to $\alpha^{\prime}$. In this way each element of $H$ yields a new action $\beta^{\prime}$ on $X$ well defined up to expected equivalence (fixing $\nu(M)$ as for 3.5). It follows from the discussion of wedge cobordisms in $[44, \S 2.5]$ that the new action $\beta^{\prime}$ on $X$ is concordant to $\beta$ in a strong sense which guarantees that the action $\beta \times 1$ on $X \times R$ is conjugate to $\beta^{\prime} \times 1$ fixing $\nu(M \times R)$. Nevertheless $\beta$ and $\beta^{\prime}$ are often inequivalent. J. Sondow has shown (unpublished) that if $(X, M)=\left(\Sigma^{n}, \Sigma^{k}\right), n \geqq 6$, $n-k \geqq 3$, is a pair of homotopy spheres, and $\alpha$ is free outside $\Sigma^{k}$, then distinct elements of $H$ give distinct actions. See [62].

4. Unknotting complements of spheres. Let $A$ be a subset of a topological space $X$. One says that $X-A$ is 1-LC at a given point $x$ in $X$ if for each neighbourhood $U$ of $x$ in $X$ there exists a neighbourhood $V \subset U$ such that every map of the circle $S^{1} \rightarrow V-A$ is homotopic in $U-A$ to a constant map.

The purpose of this section is to prove:

THEOREM 4.1 (CONJECTURED IN [12]). Suppose $\Sigma^{k}$ is a $k$-sphere topologically imbedded in $S^{n}$ so that $S^{n}-\Sigma^{k}$ is 1-LC at each point of $\Sigma^{k}$. Then, provided $n \neq 4$, $S^{n}-\Sigma^{k}$ is homeomorphic to $S^{n}-S^{k}$ where $S^{k} \subset S^{n}$ is the standard $k$-space.

COMPLEMENT 4.2. $S^{n}-\Sigma^{k}$ is in fact PL homeomorphic and diffeomorphic to $S^{n}-S^{k}$.

Discussion 4.3. (a) Notice that if $\Sigma^{k}$ is locally flat ( $\left.{ }^{13}\right)$ in $S^{n}$ and $n-k \neq 2, n \geqq 5$, then by Stallings [48], $\left(S^{n}, \Sigma^{k}\right)$ is homeomorphic to $\left(S^{n}, S^{k}\right)$, so that, in this case 4.1 adds nothing new. Conceivably 4.1 never adds anything new for there is the following basic conjecture (but see (c) below):

CONJECTURE $\left({ }^{14}\right)$. Let $X^{k}$ be an unbounded topological $k$-manifold imbedded as a closed subset of $R^{n}$. If $R^{n}-X$ is 1-LC at $X, X$ is locally flatly imbedded.

For $n=3$, this follows from Bing [1].

(b) Fox and Artin [10, §3.2] give a 2-sphere $\Sigma^{2} \subset S^{3}$ wild at one point such that each component of the complement is an open disc. At the wild point $S^{3}-\Sigma^{2}$ is shown to be not 1-LC. We conclude that the 1-LC hypothesis in 4.1 is not always necessary for the conclusion. Instead it is clearly a necessary condition for flatness of $\Sigma^{k}$ in $S^{n}, n-k \neq 2$.

$\left.{ }^{(13}\right)$ This means that for each $x \in \Sigma^{k}$ there is a neighbourhood $U$ so that $\left(U, U \cap \Sigma^{k}\right)$ is homeomorphic to $\left(R^{n}, R^{k}\right)$.

$\left({ }^{14}\right)$ Bryant and Seebeck have now (1968) proved this at least in the metastable range [59]. 
(c) In 4.1 and 4.2 in the case $n-k \geqq 3$, one can replace the assumption that $\Sigma^{k}$ is a $k$-sphere by the following: $\Sigma^{k}$ is a compact ANR of dimension $<n-1$ and of the homotopy type of $S^{k}$. These are exactly the assumptions used by the proofs below.

(d) For codimension 2 I can offer only

CONJeCtURE. $A(n-2)$-sphere $\Sigma^{n-2} \subset S^{n}$ has complement homeomorphic to $S^{n}-S^{n-2}$ if $S^{n}-\Sigma^{n-2}$ is 1-ALG at each point of $\Sigma^{n-2}$ (see [12]) and $S^{n}-\Sigma^{n-2}$ has the homotopy type of a circle.

For the proof of 4.1 we need

Lemma 4.4. Suppose $X$ is a closed set of dimension $<n-1$ in $M^{n}$, a connected unbounded n-manifold. Then $M-X$ is connected and $i_{*}: \pi_{1}(M-X) \rightarrow \pi_{1} M$ is onto. If $M-X$ is 1-LC at all points of $X, i_{*}$ is an isomorphism.

Proof of 4.4. The proof is by no means new. We give an outline and refer to [27], [8] for details. The facts about dimension that one needs are:

(1) A subset of dimension $<n$ in $R^{n}$ has no interior points.

(2) A subset of dimension $<n-1$ in $R^{n}$ cannot disconnect $R^{n}$ (Hurewicz and Wallman [20, Theorem IV 4, p. 48]).

From (2) it follows that $M-X$ is (path) connected.

To establish that $i_{*}$ is onto one shows using (1) and (2) that any loop in $M$ can be moved slightly to miss $X$ (cf. [27, p. 329]).

To establish that $i_{*}$ is injective, one has to show that any map $f:\left(D^{2}, b D^{2}\right) \rightarrow$ $(M, M-X)$ can be changed on int $D^{2}$ to give a map into $M^{n}-X$. Using (1) and (2) again, one can, for any triangulation of $D^{2}$ replace $f$ by a map $g$ of the 1-skeleton of $D^{2}$ into $M-X$ such that $g$ is near $f$ and $g\left|b D^{2}=f\right| b D^{2}$. If the triangulation of $D^{2}$ is sufficiently fine, and $g$ is sufficiently near $f$, the assumption that $M-\bar{X}$ is 1-LC at all points of $X$ guarantees that $g$ extends to a map $D^{2} \rightarrow M-X$. For details see [27, p. 335] or [8, Theorem 2].

Proof of 4.1. The case $n-k=2$ does not occur since $S^{n}-\Sigma^{n-2}$ cannot be 1-LC at $\Sigma^{k}$ for homological reasons [58].

For $n \leqq 3$ one can even conclude that $\left(S^{n}, \Sigma^{k}\right)$ its homeomorphic to $\left(S^{n}, S^{k}\right)$-see Bing [1]. For $n \geqq 5$ and $k=n-1$ the result was proved by D. R. McMillan in $[27, \S 3]$.

There remains for us to prove 4.1 when $n \geqq 5$ and $n-k \geqq 3$. As $\Sigma^{k}$ is a retract of a neighbourhood, for any connected open $U \supset \Sigma^{k}$, there exists a connected open $V \supset \Sigma^{k}$ in $U$ such that $V \subseteq U$ is homotopic in $U$ to a retraction onto $\Sigma^{k}$. Since $\pi_{1}\left(\Sigma^{k}\right)=1$, we know $\pi_{1}(V) \rightarrow \pi_{1}(U)$ has image 1 . Then by $4.4, U-\Sigma^{k}$ and $V-\Sigma^{k}$ are connected and $\pi_{1}\left(V-\Sigma^{k}\right) \rightarrow \pi_{1}\left(U-\Sigma^{k}\right)$ has image 1 . It follows directly that $S^{n}-\Sigma^{k}$ has one end, $\pi_{1}$ is essentially constant at $\infty$ and $\pi_{1}(\infty)=1$.

By 4.4 again, $S^{n}-\Sigma^{k}$ is simply connected. Alexander duality says that it has the integral homology of $S^{j}, j=n-k-1$. Hence it has the homotopy type of $S^{j}$. 
According to Irwin [56] there exists a locally flat PL imbedding $f: S^{j} \rightarrow S^{n}-\Sigma^{k}$ that is a homotopy equivalence. As $\pi_{1}$ is well-behaved at $\infty$, 2.1 says $S^{n}-\Sigma^{k}$ is an open regular neighbourhood of $f\left(S^{j}\right)$. As $f\left(S^{j}\right)$ is unknotted in $S^{n}$ [57], any open regular neighbourhood is PL homeomorphic to $S^{j} \times R^{n-j}$ and hence to $S^{n}-S^{k}$.

Proof of Complement 4.2. Since there is a unique DIFF or PL structure on $S^{n}-S^{k}, n \leqq 3$, [32] and on $S^{n}-S^{n-1}, n \geqq 5$, [46], we can assume $n \geqq 5$, and $n-k \geqq 3$. Then the proof of 4.1 established a PL homeomorphism $S^{j} \times R^{n-j} \rightarrow S^{n}-\Sigma^{k}$. The Cairns-Hirsch theorem [13, Theorem 2.5] shows that $S^{n}-\Sigma^{k}$ is differentiably a product neighbourhood of some smooth $S_{0}^{j} \subset S^{n}-\Sigma^{k}$, which, under a $C^{1}$ triangulation, is PL equivalent to $S^{j}$, but unfortunately need not be diffeomorphic to $S^{j}$. Nevertheless any product neighbourhood of $S_{0}^{j}$ in $S^{n}$ is diffeomorphic to $S^{j} \times R^{n-j}$ by [14, Theorem 7].

Added in proof (April 28, 1969). To bring this article up to date, I add

Open Collar Theorem 1.6 (TOP). The version of the Open Collar Theorem 1.6 for TOP (=topological) manifolds is valid in dimensions $\geq 5$.

This is not an empty generalization since many topological manifolds admit no PL manifold structure, even stably [64].

For dimensions $\geqq 6$ the proof in the text can now be carried out successfully in the category of TOP manifolds because recent work of Kirby and the author [64], [45] has established the needed TOP handlebody methods in dim $\geqq 6$. Newman's topological engulfing replaces Stallings' (cf. [42]).

For dimension 5, it seems necessary (at the moment) to reinforce the "pure engulfing" approach of [42] with results of [64]. My article [42] was a sequel to this one; it established $[42, \$ 4.1]$ that 1.6 (TOP) is true in case either (a) $W$ is 2-connected or (b) $b W \times R$ admits a PL structure. The assumption (a) or (b) serves in [42, §4] only to provide an open subset $U_{2}$ of $W(W$ is $M$ in [42, §4]) such that

(i) $U_{2}$ admits a PL structure.

(ii) The closure $\bar{U}_{2}$ is a locally flat submanifold of codimension zero.

(iii) $\pi_{i}\left(W-U_{2}, \bar{U}_{2}-U_{2}\right)=0, i \leq 2$.

Condition (iii) is the one that I should have cited on line 5 of [42, $\$ 4$, p. 256]. (Also read "contractible manifold" on line 17.)

Here is a method for constructing such a $U_{2}$ in case $\operatorname{dim} W \geq 5$. Triangulate a topological normal closed disc bundle $T$ to int $W$ in euclidean space. This is possible because $T$ is parallelizable [64, (I)]. Deform the projection $p: T \rightarrow \operatorname{int} W$, restricted to the 2-skeleton $T^{(2)}$, to a map $p^{\prime}: T^{(2)} \lessgtr V$ such that $V$ is open in int $W$ and has a PL structure $\Sigma$ making $p^{\prime}$ a proper PL embedding. The reader can construct $p^{\prime}, V$ and $\Sigma$ using [64, (I), (II)] and finite induction over (dim $W+1)$ coordinate charts covering int $W[38, \S 3.6]$. Finally define $U_{2}$ to be the interior of a regular neighborhood of $p^{\prime} T^{(2)}$ in $V$. 


\section{REFERENCES}

1. R. H. Bing, A surface is tame if its complement is 1-ULC, Trans. Amer. Math. Soc. 101 (1961), 294-305.

2. W. Browder, Structures on $M \times R$, Proc. Cambridge Philos. Soc. 61 (1965), 337-345.

3. W. Browder, J. Levine and G. R. Livesay, Finding a boundary for an open manifold, Amer. J. Math. 87 (1965), 1017-1028.

4. E. Connel, D. Montgomery and C. T. Yang, Compact groups in $E^{n}$, Ann. of Math. (2) 80 (1964), 94-103.

5. —__ Ann. of Math. 81 (1965), 194 (correction to [4]).

6. Sylvia de Christ, El transfer en los grupos de Whitehead, Notices Amer. Math. Soc. 13 (1966), 851.

7. A. Douady, Séminaire H. Cartan, 1961-1962, Secrétariat Mathématique, Paris, Exposé 1.

8. S. Eilenberg and R. L. Wilder, Uniform local connectedness and contractibility, Amer. J. Math. 64 (1942), 613-622.

9. D. Epstein, The degree of a map, Proc. London Math. Soc. (3) 16 (1966), 369-383.

10. R. H. Fox and E. Artin, Some wild cells and spheres in 3-dimensional space, Ann. of Math. (2) 49 (1948), 979-990.

11. A. Haefliger and C. T. C. Wall, Piecewise-linear bundles in the stable range, Topology 4 (1965), 209-214.

12. J. P. Hempel and D. R. McMillan, Locally nice embeddings of manifolds, Amer. J. Math. 88 (1966), 1-19.

13. M. Hirsch, On combinatorial submanifolds of differentiable manifolds, Comment. Math. Helv. 36 (1961), 108-111.

14. - Smooth regular neighborhoods, Ann. of Math. (2) 76 (1962), 524-530.

15. - Obstruction theories for smoothing manifolds and maps, Bull. Amer. Math. Soc. 69 (1963), 352-356.

16. — On tangential equivalence of manifolds, Ann. of Math. (2) 83 (1966), 211-217.

17. - On normal microbundles, Topology 5 (1966), 229-240.

18. J. F. P. Hudson and E. C. Zeeman, On combinatorial isotopy, Inst. Hautes Études Sci. Publ. Math., No. 19 (1964), 69-94.

19. - On regular neighbourhoods, Proc. London Math. Soc. (3) 14 (1964), 719-745.

20. W. Hurewicz and H. Wallman, Dimension theory, Princeton Univ. Press, Princeton, N. J., 1948.

21. K. Jänich, Differenzierbare Mannigfaltigkeiten mit Rand als Orbiträume differenzierbarer G-Mannigfaltigkeiten ohne Rand, Topology 5 (1966), 301-320.

22. M. Kervaire, Le théorème de Barden-Mazur-Stallings, Comment. Math. Helv. 40 (1965), 31-42.

23. B. Mazur, Stable equivalence of differentiable manifolds, Bull. Amer. Math. Soc. 67 (1961), 377-384.

24. - Relative neighbourhoods and the theorems of Smale, Ann. of Math. (2) 77 (1963), 232-249.

25. - The method of infinite repetition in pure topology. I, Ann. of Math. (2) 80 (1964), 201-226.

26. - The method of infinite repetition in pure topology. II. Stable applications, Ann. of Math. (2) 83 (1966), 387-401.

27. D. R. McMillan, Jr., A criterion for cellularity in a manifold, Ann. of Math. (2) 79 (1964), 327-337.

28. J. Milnor, Two complexes which are homeomorphic but combinatorially distinct, Ann. of Math. (2) 74 (1961), 575-590. 
29. J. Milnor., Differential structures, Lectures notes, Princeton University, Princeton, N. J., 1961 .

30. - Morse theory, Ann. of Math. Studies, No. 51, Princeton Univ. Press, Princeton, N. J., 1963.

31. - Microbundles; Part I, Topology 3, Suppl. 1 (1964), 53-80.

32. Lectures on the h-cobordism theorem, Princeton Mathematical Notes, Princeton Univ. Press, Princeton, N. J., 1965.

33. — Whitehead torsion, Bull. Amer. Math. Soc. 72 (1966), 359-426.

34. J. Munkres, Differential isotopies of the 2-spheres, Michigan Math. J. 7 (1960), 193-197.

35. — Obstructions to extending diffeomorphisms, Proc. Amer. Math. Soc. 15 (1964), 297-299.

36. M. H. A. Newman and J. H. C. Whitehead, On the group of a certain linkage, Quart. J. Math. (2) 8 (1937), 41-21.

37. M. H. A. Newman, Boundaries of ULC sets in Euclidean space, Proc. Nat. Acad. Sci. U.S.A. 34 (1948), 193-196.

38. R. Palais, "Slices and equivariant imbeddings," pp. 101-115 in Seminar on transformation groups by A. Borel, Ann. of Math. Studies, No. 46, Princeton Univ. Press, Princeton, N. J., 1960.

39. A. Scott, Infinite regular neighborhoods, J. London Math. Soc. 42 (1967), 245-253.

40. L. Siebenmann, Doctoral dissertation, Princeton University, 1965, Dissertation Abstracts, 27 (June 1966), pp. 2044-2045 (Order No. 66-5012), University Microfilms Ltd., 300 N. Zeeb Rd., Box 1346 Ann Arbor, Michigan.

41. W. B. R. Lickorish and L. Siebenmann, Regular neighborhoods and the stable range, Trans. Amer. Math. Soc. 139 (1969), 207-230.

42. L. Siebenmann, On detecting euclidean space homotopically among topological manifolds, Invent. Math. 6 (1968), 245-261.

43. - (version of [40] to appear).

44. L. Siebenmann and J. Sondow, Some homeomorphic sphere pairs that are combinatorially distinct, Comment. Math. Helv. 41 (1966-67), 261-272.

45. L. Siebenmann, Remarks on topological manifolds, Notices Amer. Math.Soc. 16(1969), 698.

46. J. Stallings, The piecewise-linear structure of euclidean space, Proc. Cambridge Philos. Soc. 58 (1962), 481-488.

47. —- On infinite processes, Differential and Combinatorial Topology, Princeton Univ.

Press, Princeton, N. J., 1965, pp. 245-254.

48. - On topologically unknotted spheres, Ann. of Math. (2) 77 (1963), 490-503.

49. N. Steenrod, Topology of fiber bundles, Princeton Univ. Press, Princeton, N. J., 1951.

50. T. Stewart, Fixed point sets and equivalence of differentiable transformation groups, Comment. Math. Helv. 38 (1963), 6-13.

51. J. B. Wagoner, Piecewise linear handlebody theory, Doctoral dissertation, Princeton University, Princeton, N.J., 1966, Appendix I, pp. 128-152.

52. C. T. C. Wall, Finiteness conditions for CW-complexes. II, Proc. Roy. Soc. Ser. A 295 (1966), 129-139.

53. E. C. Zeeman, On the dunce hat, Topology 2 (1964), 341-358.

54. J. Stallings, On polyhedral topology, Lecture notes on mathematics, Tata Inst. of Fundamental Research, Bombay, 1968.

55. M. M. Cohen, A general theory of relative regular neighborhoods, Trans. Amer. Math. Soc. 136 (1969), 189-229.

56. M. C. Irwin, Embeddings of polyhedral manifolds, Ann. of Math. (2) 82 (1965), 1-14.

57. E. C. Zeeman, Unknotting combinatorial balls, Ann. of Math. (2) 78 (1963), 501-526. 
58. P. Alexandroff, On local properties of closed sets, Ann. of Math. (2) 36 (1935), 1-35.

59. J. L. Bryant and C. L. Seebeck, Locally nice embeddings in codimension 3, Bull. Amer. Math. Soc. 74 (1968), 378-380.

60. N. H. Kuiper and D. Burghelia, Hilbert manifolds, Ann. of Math. (to appear).

61. E. H. Spanier, Algebraic topology, McGraw-Hill, New York, 1966.

62. M. Rothenberg and J. Sondow, Non-linear smooth representations of compact Lie groups, Preprint, Univ. of Chicago, 1969.

63. J. F. P. Hudson, Piecewise linear topology, Benjamin, New York, 1969.

64. R. C. Kirby and L. Siebenmann, On the triangulation of manifolds and the Hauptvermutung, Bull. Amer. Math. Soc. 75 (1969), 741-748.

65. S. Lang, Tangential homotopy equivalence, Séminaire Bourbaki, 1961.

\section{UNIVERSITY OF PARIS,}

91-Orsay, France 